\title{
Investigating the Role of Surface Depletion in Governing Electron Transfer Events in

\author{
Colloidal Plasmonic Nanocrystals
}

\author{
Bharat Tandon $^{\mathbb{I \dagger}}$, Sofia A. Shubert-Zuleta ${ }^{\dagger \ddagger}$, Delia J. Milliron ${ }^{\mathbb{I f *} *}$ \\ ${ }^{"}$ McKetta Department of Chemical Engineering, University of Texas at Austin, \\ Austin, Texas 78712-1589, United States \\ ${ }^{*}$ Department of Chemistry, University of Texas at Austin, Austin, Texas 78712-
}

\author{
1589, United States
}

\begin{abstract}
:
Doped metal oxide nanocrystals (NCs) attract immense attention because of their ability to exhibit a localized surface plasmon resonance (LSPR) that can be tuned extensively across the infrared region of the electromagnetic spectrum. LSPR tunability triggered through compositional and morphological changes during the synthesis (size, shape and doping percentage) is becoming wellestablished while the principles underlying dynamic, post-synthetic modulation of LSPR are not as well understood. Recent reports have suggested that the presence of a depletion layer on the NC surface may be instrumental in governing the LSPR modulation of doped metal oxide NCs. Here, we employ post-synthetic electron transfer to colloidal Sn-doped $\operatorname{In}_{2} \mathrm{O}_{3} \mathrm{NCs}$ with varying size and Sn doping concentration to investigate the role of the depletion layer in LSPR modulation. By measuring the maximum change in LSPR frequency after NC reduction, we determine that a large initial volume fraction of the depletion layer in NCs results in a broad modulation of the LSPR energy and intensity. Utilizing a mathematical Drude fitting model, we track the changes in the electron density and the depletion layer volume fraction throughout the chemical doping process, offering fundamental insight into the intrinsic NC response resulting from such electron transfer events. We observe that the maximum change in electron density that can be induced through chemical doping is independent of Sn concentration, and subsequently, the maximum total electron density in the presence of excess reductant is independent of the NC diameter and is dependent only on the as-synthesized Sn doping concentration. This study establishes the central role that surface depletion plays in the electronic changes occurring in the NCs during post-synthetic doping and the results will be instrumental in advancing the understanding of optical and electrical properties of different colloidal plasmonic NCs.
\end{abstract}

\section{Introduction:}

Degenerately doped semiconductor nanocrystals (NCs) and in particular, doped metal oxide NCs have emerged as an important class of materials in the last decade for their ability to sustain localized surface plasmon resonance (LSPR) in the mid- to near-infrared region of the electromagnetic spectrum. LSPR, which arises due to the resonant oscillations of carriers with 
electromagnetic radiation, has predominantly been studied in noble metals such as Au and Ag. ${ }^{1-4}$ Already in that context, LSPR has found a variety of applications such as photothermal therapy, ${ }^{5}$ photovoltaics, ${ }^{6}$ electrochromic windows, ${ }^{7-9}$ sensing ${ }^{10}$ and surface-enhanced infrared absorption spectroscopy. ${ }^{1}$ Unlike noble metals whose LSPR remains restricted to a narrow window in the visible region, doped metal oxide NCs offer an advantage in that their LSPR that can be tuned across a wide spectral range in the infrared region. While in noble metals, infrared resonance requires large sizes or highly anisotropic shapes, in doped semiconductors this tunability can also be achieved by merely altering the free carrier density in the NCs (see equation 1).

$$
\omega_{p}=\sqrt{\frac{n_{e} e^{2}}{\varepsilon_{o} m^{*}}}
$$

where $\omega_{p}$ is the bulk plasma frequency that governs the LSPR, $n_{e}$ is the carrier density, $e$ is the elementary charge, $\varepsilon_{o}$ is the dielectric constant of free space, and $m^{*}$ is the effective carrier mass. ${ }^{4}$

While many reports have focused on modifying carrier density through intrinsic doping (vacancy generation) and extrinsic doping (aliovalent doping), a distinctive advantage of LSPR in doped semiconductor NCs is the opportunity for substantial dynamic post-synthetic modulation of the carrier density. ${ }^{7,}$ 11-15 This post-synthetic modification of the carrier density can be achieved using an external source of carriers, such as an electrochemical potential, light-activated reduction, or a chemical redox agent. ${ }^{7,16-19}$

For instance, addition of an aliovalent dopant during $\operatorname{In}_{2} \mathrm{O}_{3} \mathrm{NC}$ synthesis leads to degenerate doping of the semiconductor and consequently, doped $\mathrm{In}_{2} \mathrm{O}_{3} \mathrm{NCs}$ exhibit LSPR in the near-infrared region. ${ }^{20-24}$ The electrons provided by post-synthetic doping are expected to have a chemical potential above the Fermi level potential. However, due to the presence of surface states, the Fermi level is pinned to the surface potential, triggering the formation of a depletion (low carrier density) region at the surface of the NC. ${ }^{25}$ Therefore, modulation of surface sensitive properties like LSPR, catalysis, sensing and electrical conductivity in degenerately doped materials such as doped $\operatorname{In}_{2} \mathrm{O}_{3}$ NCs becomes inherently dependent on the presence of this surface depletion layer. ${ }^{25-29}$

Indeed, studying electrochemical modulation of LSPR in thin films of Sn-doped $\operatorname{In}_{2} \mathrm{O}_{3}\left(\mathrm{Sn}_{\left.\mathrm{In}_{2} \mathrm{O}_{3}\right)}\right.$ NCs showed that the extent to which the LSPR shifts during charging is controlled by the fraction of the NC volume occupied by the depletion layer at the surface, which depends on both NC size and Sn dopant concentration. ${ }^{30}$ However, in electrochemically charged thin films, net LSPR shift of doped metal oxide NCs is dependent on a competition between depletion effects and changes in plasmonic coupling between NCs, which makes it difficult to precisely identify the contribution of depletion effects in controlling LSPR modulation of doped metal oxide NCs. ${ }^{19}$ Performing spectroelectrochemical investigations on colloidal dispersions of polymer-wrapped Sn-doped $\mathrm{In}_{2} \mathrm{O}_{3} \mathrm{NCs}$ circumvents LSPR coupling effects, but it's difficult to stabilize colloidal dispersions over a wide range of electrochemical conditions, limiting the range of information that has been gleaned by this approach. ${ }^{31}$ Thus, we sought a method of post-synthetic doping in colloidal dispersions of $\mathrm{Sn}: \mathrm{In}_{2} \mathrm{O}_{3} \mathrm{NCs}$ that maintained NC stability, in order to isolate the effects of the depletion layer on the LSPR modulation. In that respect, chemical doping can be performed on 
stable colloidal dispersions of NCs and with an appropriate reducing (oxidizing) reagent, it also enables facile transfer of electrons to (from) the NCs. Thus, to deconvolute the contribution of depletion effects in governing the extent of LSPR modulation, we carried out chemical doping on $\mathrm{Sn}: \mathrm{In}_{2} \mathrm{O}_{3} \mathrm{NCs}$ of varying sizes and doping concentrations. In addition, this also allowed us to systematically investigate the effects of chemical doping on LSPR of NCs with different size and doping percentage to compare with earlier electrochemical studies carried out on similar NCs. ${ }^{30-31}$

Chemical doping has been extensively utilized in dispersions of metal oxide NCs to reveal information about electronic energy levels and their relationship to their chemical environment and to other NCs. ${ }^{32-36}$ The optical band gap energy and magnitude of near-infrared absorption due to free electrons increases in undoped $\mathrm{ZnO}$ NCs when decamethylcobaltocene $\left(\mathrm{CoCp}^{*}\right)$ is added to a dispersion, signifying electron transfer to the $\mathrm{ZnO} \mathrm{NCs} .{ }^{33} \mathrm{CoCp}_{2}{ }_{2}$ is an ideal reductant to use because it is highly soluble in nonpolar solvents typically used to disperse NCs. CoCp* ${ }_{2}$ has a high standard reduction potential of $-1.95 \mathrm{~V} \mathrm{vs} \mathrm{Fc}^{+/ 0}$ (ferrocenium/ferrocene redox pair), high enough to reduce $\mathrm{ZnO}$ and presumably other metal oxide NCs. ${ }^{19}$ Further, the magnitude of electron transfer from CoCp $*_{2}$ to NCs is increased substantially by addition of an acid to facilitate proton-coupled electron transfer. ${ }^{20}$ Given that $\mathrm{ZnO}$ and $\mathrm{In}_{2} \mathrm{O}_{3}$ have similar band gap energy and band-edge positions, ${ }^{37}$ we hypothesized that $\mathrm{CoCp}^{*} 2$ should also be able to spontaneously reduce $\mathrm{Sn}: \mathrm{In}_{2} \mathrm{O}_{3}$ NCs.

Here, results show that volumetric additions of $\mathrm{CoCp}^{*} 2$ to colloidal dispersions of $\mathrm{Sn}: \mathrm{In}_{2} \mathrm{O}_{3} \mathrm{NCs}$ indeed causes a systematic blue-shift of the LSPR, while also retaining the colloidal stability of the NCs. With an increase in NC size or Sn doping percentage, we observed a progressive decrease in the maximum LSPR shift achieved, which is in excellent correlation with the observations previously made for $\mathrm{Sn}: \mathrm{In}_{2} \mathrm{O}_{3} \mathrm{NC}$ films. ${ }^{30}$ Notably, the magnitude of maximum LSPR shift achievable through chemical doping is significantly higher than that achieved in films or even with solution phase electrochemical doping, for NCs of similar size and Sn doping percentage. ${ }^{31}$ Through a recently developed Drude fitting model that accounts for depletion, ${ }^{38}$ we demonstrate that electron transfer from $\mathrm{CoCp}_{2}{ }_{2}$ to $\mathrm{NCs}$ during chemical titration results in a decrease in the volume fraction of the depletion region. The plasmonic core of the NC expands and the concentration of electrons in that core region increases. We rationalize the trends in the maximum achievable LSPR shift across a range of NC diameters and doping concentrations by analyzing changes in the depletion region volume fraction and using quantitative analysis, we track the changes in the electron density throughout the chemical reduction process.

\section{Results and Discussion:}

The extent of the depletion layer is determined by synthetically controlled physical attributes such as the doping percentage, radial dopant distribution, size and shape of the NCs. Therefore, synthetic conditions must be optimized to yield NCs with precise control over these parameters to set the stage for a reliable quantitative assessment of electron transfer. Thus, by adapting a continuous injection procedure developed by the Hutchison group, ${ }^{39-40}$ we first synthesized highly monodisperse spherical Sn: $\operatorname{In}_{2} \mathrm{O}_{3} \mathrm{NCs}$ with different sizes and Sn doping percentages and uniform dopant distribution. More precisely, by adjusting the injection volume and ratio of the metal ion precursors (see experimental section for more details), NCs with average diameter between 8-21 
$\mathrm{nm}$ and Sn doping percentage between $4.5-13 \%$ were synthesized. A summary of synthesized NCs listing the doping percentages obtained from inductively coupled plasma atomic emission spectroscopy (ICP-AES) and the average diameter from scanning transmission electron microscopy (STEM) is provided in Table 1.

Table 1: Summary of the doping percentage determined by ICP-AES and diameters determined by STEM of different $\mathrm{Sn}: \operatorname{In}_{2} \mathrm{O}_{3}$ NCs employed for chemical titration experiments.

\begin{tabular}{|c|c|c|}
\hline Nominal Sn \% & Actual Sn \% & Diameter (nm) \\
\hline \multirow{4}{*}{$4.5 \%$} & $3.9 \pm 0.02$ & $8.3 \pm 0.7$ \\
\hline & $4.3 \pm 0.02$ & $12.5 \pm 0.6$ \\
\hline & $4.3 \pm 0.05$ & $17.0 \pm 2.0$ \\
\hline & $4.3 \pm 0.03$ & $20.7 \pm 1.7$ \\
\hline \multirow{5}{*}{$6 \%$} & $6.1 \pm 0.04$ & $7.6 \pm 0.9$ \\
\hline & $5.8 \pm 0.02$ & $11.4 \pm 1.0$ \\
\hline & $5.8 \pm 0.01$ & $12.7 \pm 1.0$ \\
\hline & $6.8 \pm 0.03$ & $17.3 \pm 1.4$ \\
\hline & $7.2 \pm 0.17$ & $21.6 \pm 3.2$ \\
\hline \multirow{3}{*}{$10 \%$} & $9.8+0.08$ & $7.3 \pm 1.6$ \\
\hline & $10.0 \pm 0.03$ & $15.3 \pm 0.8$ \\
\hline & $9.6 \pm 0.01$ & $22.3 \pm 1.5$ \\
\hline & & \\
\hline \multirow{3}{*}{$13 \%$} & $13.1 \pm 0.07$ & $8.8 \pm 0.7$ \\
\hline & $13.2 \pm 0.02$ & $12.2 \pm 1.9$ \\
\hline & $11.7 \pm 0.13$ & $22.2 \pm 1.1$ \\
\hline
\end{tabular}

All NCs exhibit spherical morphology with low polydispersity $(\sim 10 \%)$ in their diameter (Figure 1a-d and S1-S3). Across the sample series, an increase in the NC diameter leads to a progressive increase of the LSPR peak energy, because of higher dopant activation and a decrease in surface scattering, both of which contribute to a higher electron density (Figure 1e and S4). ${ }^{28,41-42}$ For a given size, the LSPR peak shifts to higher frequency as Sn doping percentage increases, owing to a progressively higher electron concentration (Figure 1f and S4). ${ }^{20,28}$

To study the effects of depletion on LSPR modulation through chemical doping, the as-synthesized NCs were dispersed in anhydrous toluene/tetrahydrofuran (THF) (1:1 ratio) mixture anaerobically.

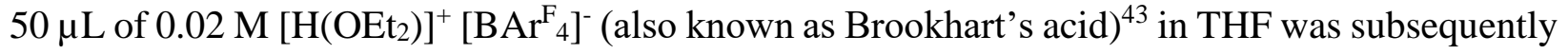
added to the NC dispersion and this mixture was titrated with $5 \mu \mathrm{L}$ aliquots of $0.02 \mathrm{M} \mathrm{CoCp} *_{2}$ solution in THF : toluene (1:1) while extinction measurements were performed continuously through an optical fiber assembly (see experimental section for details). 

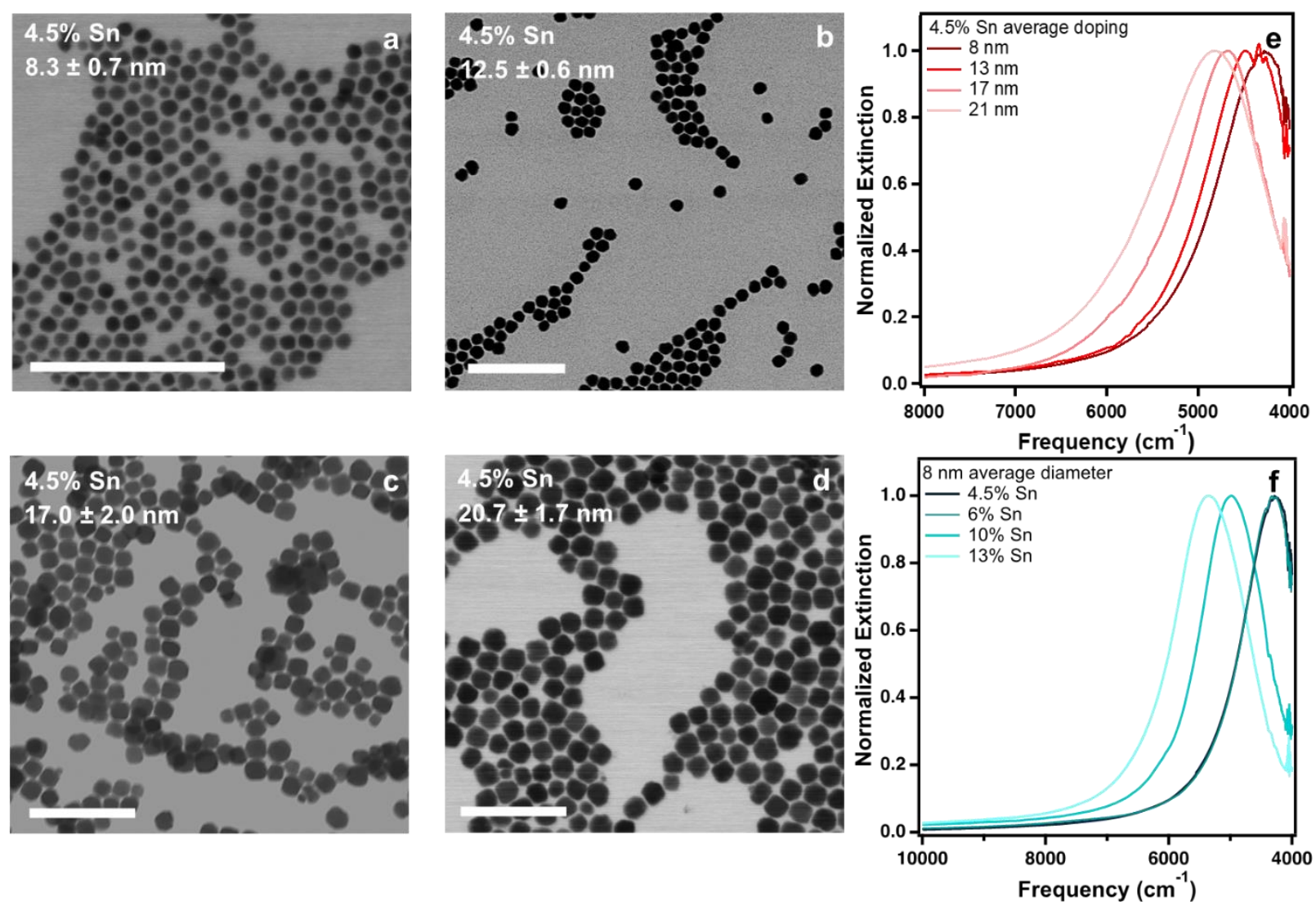

Figure 1: (a-d) STEM images of $4.5 \% \mathrm{Sn}: \mathrm{In}_{2} \mathrm{O}_{3} \mathrm{NCs}$ with spherical morphology and different diameters. All scale bars are $100 \mathrm{~nm}$ (e) normalized extinction spectra for $4.5 \% \mathrm{Sn}: \mathrm{In}_{2} \mathrm{O}_{3} \mathrm{NCs}$ with different diameters (f) normalized extinction spectra for different $\mathrm{Sn}: \mathrm{In}_{2} \mathrm{O}_{3} \mathrm{NCs}$ with $8 \mathrm{~nm}$ as average diameter.

Addition of $\left[\mathrm{H}\left(\mathrm{OEt}_{2}\right)\right]^{+}\left[\mathrm{BAr}_{4}\right]^{-}$acid solution to the $\mathrm{NC}$ dispersion preserves the original LSPR frequency and peak width (Figure S5), which suggests that addition of protons doesn't deteriorate the colloidal stability of NCs or initiate any electron transfer. This observation is consistent with the previous assessment that proton-coupled electron transfer occurs only upon addition of a reducing agent such as $\mathrm{CoCp}_{2}^{*}$, to $\left.\left[\mathrm{H}_{(\mathrm{OEt}}\right)\right]^{+}\left[\mathrm{BAr}_{4}^{-}\right]^{-}$, likely through intercalation of chargebalancing $\mathrm{H}^{+}$ions in the NCs. ${ }^{32-33}$

Subsequent addition of $5 \mu \mathrm{L}$ aliquots of $0.02 \mathrm{M} \mathrm{CoCp}_{2}{ }_{2}$ to a mixture of $\mathrm{NCs}$ and $\left[\mathrm{H}\left(\mathrm{OEt}_{2}\right)\right]^{+}$ $\left[\mathrm{BAr}_{4}{ }_{4}^{-}\right.$acid causes a systematic shift of the LSPR energy to higher wavenumbers and an increase of the LSPR extinction even while the concentration of the NCs is being diluted (Figure 2a). Both observations suggest that there is a transfer of electrons from CoCp* ${ }_{2}$ to the $\mathrm{Sn}_{2} \operatorname{In}_{2} \mathrm{O}_{3}$ NCs which increases the electron density and overall electron population and, thus, the increased LSPR energy and extinction, respectively. The use of $\mathrm{CoCp}_{2}$ as a reducing agent, the mechanism of protoncoupled electron transfer, and the role of $\left[\mathrm{BAr}_{4}\right]^{-}$as a counter ion have been elucidated in previous reports ${ }^{32-33}$ and won't be further described in the current manuscript. 

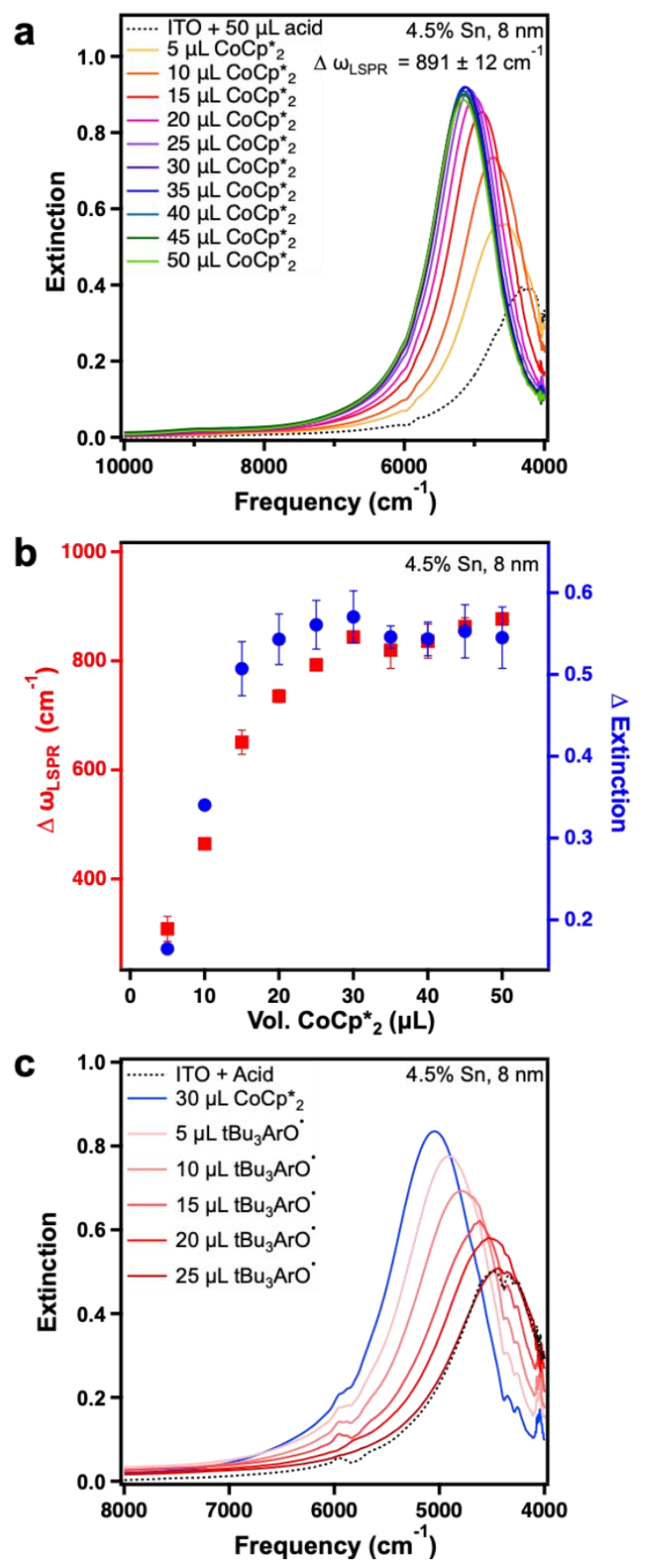

Figure 2: Representative chemical reduction using $8 \mathrm{~nm}, 4.5 \% \mathrm{Sn}: \mathrm{In}_{2} \mathrm{O}_{3} \mathrm{NCs}$ (a) Evolution of the LSPR extinction spectra during volumetric additions of $\mathrm{CoCp}_{2}{ }_{2}$ to a colloidal dispersion of NCs and $50 \mu \mathrm{L} 0.02 \mathrm{M}$ Brookhart's acid (b) Variation in the LSPR peak extinction and LSPR energy with additions of $\mathrm{CoCp}_{2}$ (c) Establishing the reversibility of the chemical reduction by titrating with a mild oxidant tri-tertbutylphenoxy radical.

Both LSPR energy and extinction reach a saturation point around the same amount of added reducing agent (Figure 2 b). Further addition of $\mathrm{CoCp}_{2}{ }_{2}$ aliquots results in broadening of the LSPR 
linewidth and decrease in the LSPR extinction signifying partial loss in the colloidal stability of the NCs. This suggests that NCs have been charged beyond their thermodynamic ability to take up electrons within the conduction band and their spontaneous precipitation may be associated with other charging pathways, for example leading to a build-up of surface charge. The maximum LSPR shift throughout the manuscript is therefore reported as the difference in the LSPR energy of the saturation point and that of the original $\mathrm{NC}$ dispersion, $\Delta \omega_{L S P R}=\omega_{\text {saturated LSPR }}-$ $\omega_{\text {as-synthesized LSPR }}$. The increase in the LSPR energy can be completely reversed by the addition of an oxidizing agent such as 2,4,6-Tri-tert-butyl-phenoxy radical ${ }^{44}$ validating that the LSPR modulation is due to a change in electron density in the NCs, rather than changes in the dielectric environment, for example.
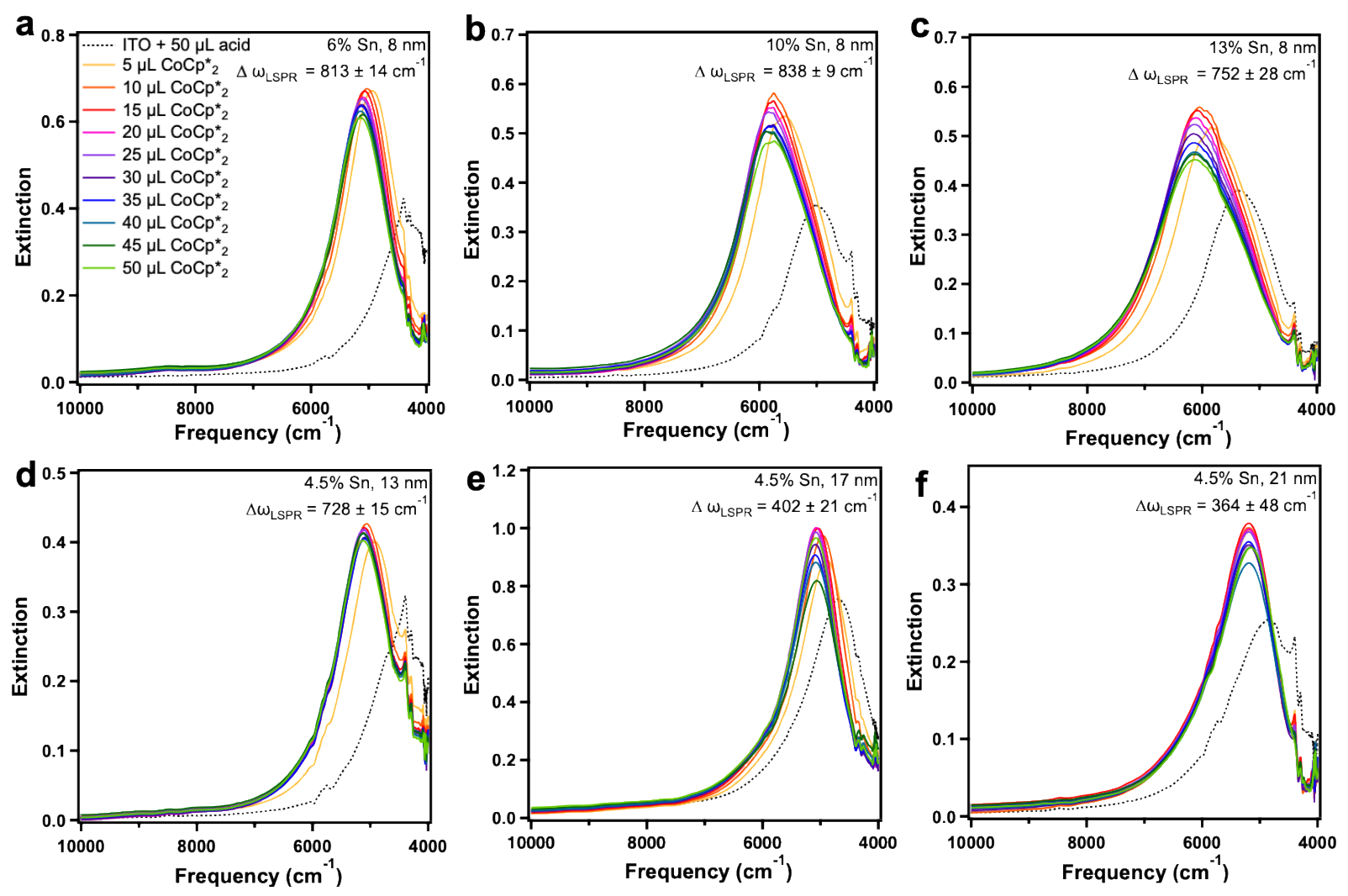

Figure 3: Effect of the addition of chemical reductant $\mathrm{CoCp}_{2}{ }_{2}$ on the LSPR extinction spectra of (a-c) $8 \mathrm{~nm} \mathrm{Sn}: \mathrm{In}_{2} \mathrm{O}_{3} \mathrm{NCs}$ with different doping percentages (d-f) $4.5 \% \mathrm{Sn}: \mathrm{In}_{2} \mathrm{O}_{3} \mathrm{NCs}$ with different $\mathrm{NC}$ diameters.

As expected, the maximum LSPR shift for a given NC composition (size, dopant concentration) is independent of the concentration in dispersion over a wide range (Figure S6). This consistent saturation behavior suggests that $\mathrm{CoCp}_{2}{ }_{2}$ makes an excess of electrons available, always exceeding the maximum number that can be added to the NCs. Therefore, the maximum LSPR frequency shift can be compared across samples, regardless of differences in NC concentration. Furthermore, to confirm reproducibility of the maximum LSPR shift values, each NC composition was measured in triplicate under the same conditions and the average of their maximum LSPR shift values are reported with uncertainties obtained from statistical analysis. 
Although addition of $\mathrm{CoCp}_{2}{ }_{2}$ solution results in a simultaneous increase in the LSPR extinction and frequency for all samples, the maximum LSPR shift is significantly affected by the NC diameter and Sn doping concentration. To separate the effects of each of these parameters on the LSPR shift, we compare a $\mathrm{Sn}: \mathrm{In}_{2} \mathrm{O}_{3}$ doping series with consistent diameter and a size series with consistent Sn doping concentration. Increasing the Sn doping concentration while keeping the diameter nearly constant leads to a decrease in the maximum LSPR shift (Figure 2a and 3a-c). Highly doped NCs have a thin depletion layer and a plasmonic core with a high initial concentration of electrons, which changes little when extra electrons are added, thus limiting the change in LSPR frequency. ${ }^{30-31}$ Similarly, when we increase the diameter of the NCs while keeping the doping percentage almost constant, we observe a decrease in the maximum LSPR shift (Figure $2 \mathrm{a}$ and $3 \mathrm{~d}-\mathrm{f}$ ). In larger NCs, added electrons are also accommodated mostly in the depletion layer with the electron concentration in the plasmonic core remaining nearly unchanged. Conversely, in smaller, lightly doped NCs, the band bending due to depletion extends through much of the NC volume, so adding electrons in this region does significantly increase the electron concentration
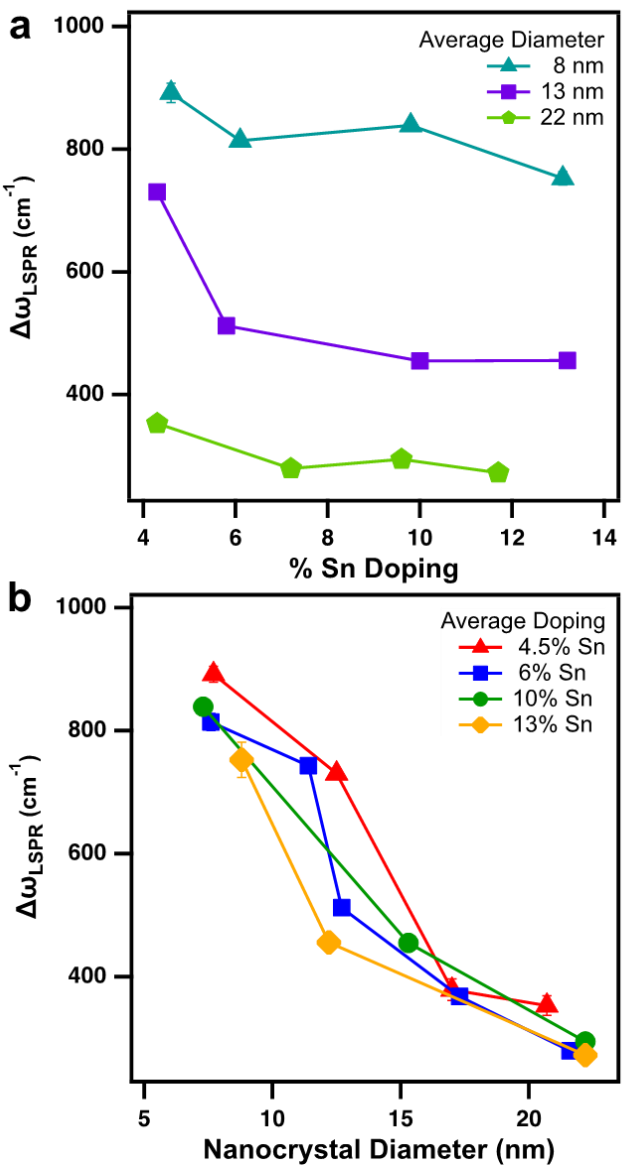

Figure 4: Summary of the maximum LSPR shift for $\mathrm{Sn}: \mathrm{In}_{2} \mathrm{O}_{3} \mathrm{NCs}$ with (a) similar average diameter but different doping percentage (b) similar doping percentage but different average diameter.

responsible for the LSPR. To summarize, increasing the NC diameter or the Sn doping concentration leads to a decrease in the depletion layer volume fraction and corresponding 
maximum LSPR shift. These trends with size and Sn doping are all in excellent correlation with prior observations regarding electrochemical charging made by Zandi et al. ${ }^{30}$

These maximum LSPR shift trends are confirmed across all other size and doping series (Figures S7-S11). For all NC diameters, the maximum LSPR shift decreases with increasing Sn doping concentration (Figure 4a). However, the maximum LSPR shift changes only moderately because at the relatively high doping concentrations studied here, the initial electron concentrations are already high, so a large change in electron population is needed to significantly shift the LSPR. Independent of the Sn doping concentration, increasing the NC diameter similarly leads to a decrease in the LSPR shift (Figure 4b). The change with NC diameter is more drastic than with dopant concentration because a small change in the NC diameter causes a large change in the volume fraction of the depletion region and ultimately the maximum LSPR shift.

While the overall trends of LSPR modulation obtained through chemical doping and electrochemical doping are qualitatively similar, on a quantitative scale, some stark differences can be observed. Inter-NC LSPR coupling becomes stronger as electrons are added in thin films, counteracting and convoluting the individual NC trends in LSPR shifts and making a clear assessment of the importance depletion effects on the LSPR of each NC challenging. Underscoring the significance of this complicating factor, the chemically induced LSPR shift of $\sim 13 \mathrm{~nm}$ NCs studied here is greater than $500 \mathrm{~cm}^{-1}$, in contrast to $226 \mathrm{~cm}^{-1}$ for $11.5 \mathrm{~nm}$ and $-191 \mathrm{~cm}^{-1}$ for $16 \mathrm{~nm}$ for electrochemical doping in thin films $10 \% \mathrm{Sn}: \mathrm{In}_{2} \mathrm{O}_{3} \mathrm{NCs} .{ }^{19,}{ }^{30}$ Therefore, chemical doping has allowed us isolate the effects of depletion layer charging on the LSPR modulation by removing inter-NC plasmon coupling as a strongly competing factor.

In fact, electrochemical doping in colloidal dispersions also eliminates inter-NC LSPR coupling and can, in principle, reveal the physics governing LSPR modulation in individual NCs. Indeed, the LSPR shifts achieved through electrochemical doping of colloidal dispersions of $\operatorname{Sn}: \mathrm{In}_{2} \mathrm{O}_{3} \mathrm{NCs}$ are greater than those obtained in thin films of similar NCs. ${ }^{31}$ However, even in colloidal dispersions, there is partial offsetting of the individual $\mathrm{NC}$ trends due to the deposition of NCs on the electrode surface. In any case, the LSPR shifts obtained through chemical doping are 30-40\% greater than those obtained through electrochemical doping of colloidal dispersions of $5 \mathrm{~nm} \mathrm{10 \%}$ $\mathrm{Sn}: \mathrm{In}_{2} \mathrm{O}_{3} \mathrm{NCs}\left(435 \mathrm{~cm}^{-1}\right){ }^{31}$ The effective applied potential through $\mathrm{CoCp}^{*}{ }_{2}$ exceeds that which could be achieved by electrochemical doping without overly destabilizing the dispersion. The reduced $\mathrm{pH}$ during chemical doping lowers the energy of the bands, facilitating the enhanced electron transfer compared to electrochemical doping. ${ }^{37}$ Thus, while electrochemical doping and chemical doping are both capable of charging dispersed NCs, chemical doping is more efficient at producing large LSPR shifts, indicating that a high electron concentration is achieved.

Although the trends in the LSPR shifts imply differences in electron concentration, to assess the number of electrons transferred and discover their spatial distribution within the NCs, we need to analyze the LSPR spectra quantitatively. Specifically, considering changes in the extinction, peak energy, and lineshape during the course of the chemical reduction we can track changes in depletion width and electron concentration in the plasmonic core. In previous reports, 'extra' electrons added by chemical or photochemical reduction have been counted by titrating with an oxidizing agent such as $\left[\mathrm{FeCp}_{2}\right]^{+},{ }^{17,} 32-33,45$ but this does not account for the distribution of those 
electrons within each NC, nor among the ensemble. Polydispersity in the NC diameter and doping percentages, and surface depletion all can significantly affect the LSPR peak energy ${ }^{38}$ Calculating changes in electron concentration while ignoring depletion, in particular, can lead to an underestimation of the electron concentration change due to the assumption that electrons occupy the total volume of the NC. Further, there exists the possibility of oxidized electrons being lost to the very trap states that form the depletion layer, which could hinder the accuracy of this method.
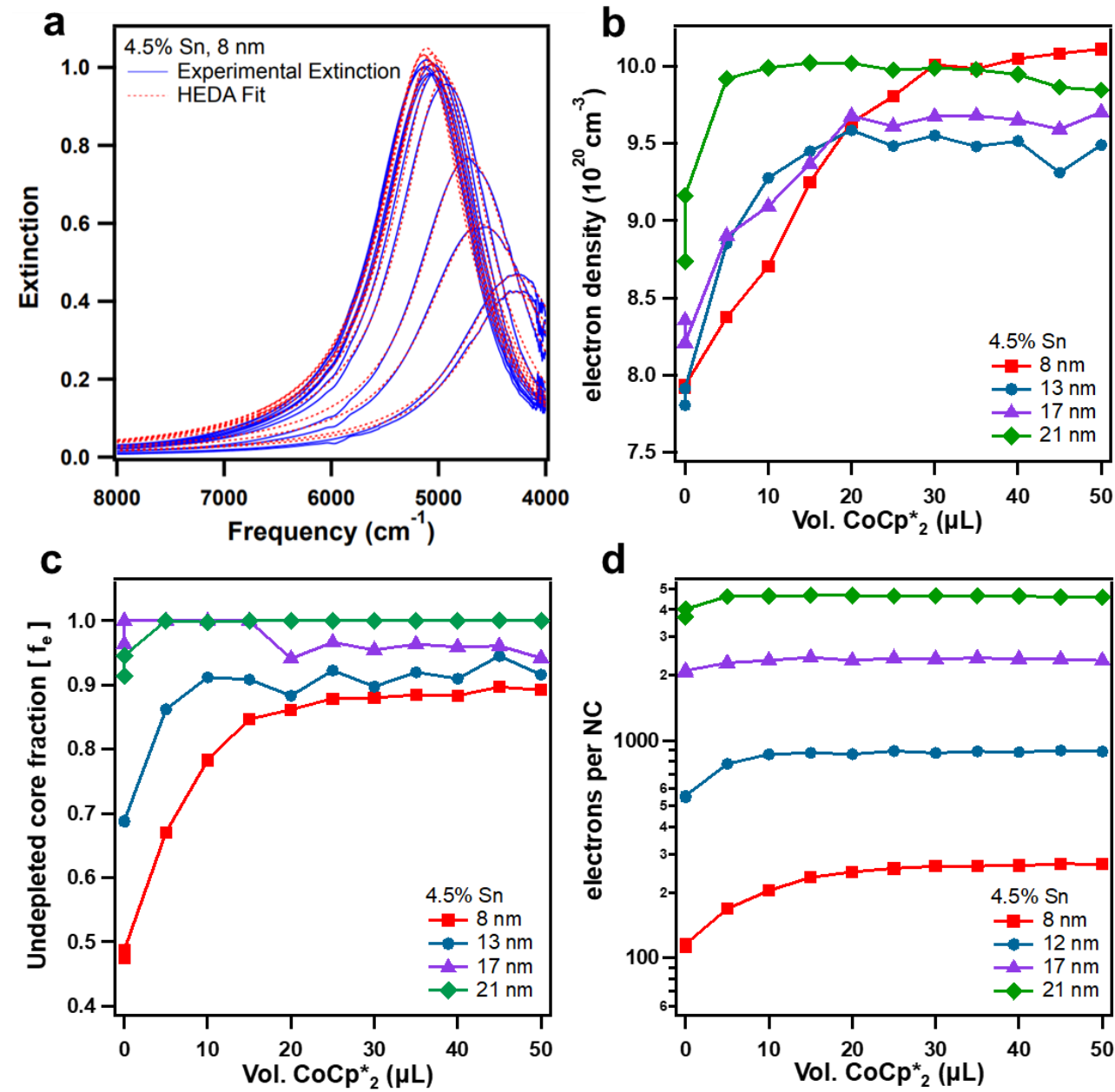

Figure 5: (a) Representative fits using HEDA model on LSPR extinction spectra of $8 \mathrm{~nm} 4.5 \%$ $\mathrm{Sn}: \mathrm{In}_{2} \mathrm{O}_{3}$ NCs. Change in the (b) electron density (c) volume fraction of the undepleted core (d) number of electrons per NC for different diameters of $4.5 \% \mathrm{Sn}: \mathrm{In}_{2} \mathrm{O}_{3} \mathrm{NCs}$. Symbols are data points, solid lines are a guide to the eye and dashed lines are least squares fits to the data points.

To overcome these limitations, we fit the LSPR spectra of our NCs with a recently developed generalization of the Drude model that takes into account heterogeneity in size and doping percentage, as well as surface depletion effects in NCs. ${ }^{38}$ The heterogeneous ensemble Drude approximation (HEDA) model takes in fixed input values from experimental data including mean $\mathrm{NC}$ radius, NC radius standard deviation, and NC volume fraction in dispersion. The model then fits the LSPR peak for electronic parameters including the electron concentration $\left(n_{e}\right)$, standard deviation in the electron concentration $\left(\sigma_{n e}\right)$, the electron accessible volume fraction $\left(f_{e}\right)$ and, the 
mean free path of the electron $\left(l_{M F P}\right)$ (see experimental section for more details on HEDA modeling). While employing spectra from an ensemble measurement, the HEDA model yields a more microscopic view of electron transfer events happening at a single NC level while deconvoluting the effects of heterogeneous peak broadening. Further, this model allows us to track depletion layer charging as a function of added reducing agent by fitting for $f_{e}$ throughout the titration, a critical aspect in correlating depletion layer volume fraction with LSPR modulation. In the development of the HEDA model, results were compared with Mie Scattering theory and physical trends with size and dopant concentration were consistent with expectations for the depletion effects. Given that the HEDA model also determines standard deviation in electron density, it is possible to verify that trends in electron density are not due to wide standard deviation or significant changes in electronic heterogeneity. We expect the HEDA model to be a reliable method for fitting the LSPR of chemically doped samples because the redox agents should modify the electron population in the NCs without substantively changing the fundamental physics governing mean free path, or other parameters built into the model.

All extinction spectra produced in the titration experiments were fit with the HEDA model, resulting in uniformly excellent fits (Figure 5a and S12). The electron concentration in the plasmonic core $\left(n_{e}\right)$ increases progressively as $\mathrm{CoCp}_{2}{ }_{2}$ is added to the $\mathrm{NC}$ dispersion (Figure $5 \mathrm{~b}$ and S13), a result of electron transfer from $\mathrm{CoCp}_{2}{ }_{2}$ to the NCs. The associated standard deviation in the electron concentration $\left(\sigma_{n e}\right)$ does not change significantly with increased addition of $\mathrm{CoCp}_{2}{ }_{2}$ (Figure S14). Concomitantly, there is also an increase in the volume fraction of the undepleted core $\left(f_{e}\right)$, so added electrons are distributed both in the core (where they increase $n_{e}$ ) and in the depletion layer (causing the core to expand) (Figure 5c). These results validate our interpretation of the increasing LSPR frequency as arising from higher $n_{e}$ in the core and reveal that the increasing extinction upon with addition of $\mathrm{CoCp}_{2}^{*}$ is due to the addition of electrons in an increasingly large plasmonic core. This is also in general agreement with previous observations on electrochemical doping of $\mathrm{Sn}: \mathrm{In}_{2} \mathrm{O}_{3} \mathrm{NCs}$, however, previous interpretations have been complicated by the presence of inter-NC plasmonic coupling, which partially obscured the increasing electron density in the plasmonic core. ${ }^{19,30-31}$ While the electron density at saturation is comparable for different sizes (Figure 5b), the maximum number of electrons per NC scales with NC volume (Figure 5d and S15), similar to previous reports. ${ }^{33,46}$ While the smallest NC (8 nm) accommodates a maximum of $\sim 270$ electrons per NC, $21 \mathrm{~nm}$ NCs are able to hold as many as 4650 electrons per NC.

In calculating the electron density and electrons per NC from the HEDA fitting results, we identify contributions to these values from the as-synthesized NCs and from the reduction process, then correlate them to the NC diameter and Sn doping concentration. For a fixed doping concentration, we find a progressive increase in the maximum number of electrons per $\mathrm{NC},\left\langle\mathrm{N}_{\max }\right\rangle$, with size, at the saturation value achieved through chemical reduction with $\mathrm{CoCp}_{2}{ }_{2}$ (Figure 6a and S16). This trend parallels the trend for the electrons per $\mathrm{NC}$ in as-synthesized $\mathrm{NCs}\left\langle\mathrm{N}_{\mathrm{sys}}\right\rangle$, as does the maximum electrons supplied through the chemical titration $\left\langle\mathrm{N}_{\text {titr }}\right\rangle$. As might be expected, a greater number of electrons can be accommodated in larger NCs, which have a higher number of dopant ions and a higher number of proton intercalating sites. This result, derived here by LSPR peak fitting, corroborates similar results previously found by electron counting methods. ${ }^{17}$ The increase 
in $\left\langle\mathrm{N}_{\max }\right\rangle$ is approximately in proportion to the $\mathrm{NC}$ volume, so the maximum electron density $\left\langle\mathrm{n}_{\max }>\right.$ after chemical reduction with $\mathrm{CoCp}_{2}{ }_{2}$ is independent of the NC diameter and seems to be fixed around $\sim 10^{21} \mathrm{~cm}^{-3}$ for $4.5 \% \mathrm{Sn}$ doping (Figure $6 \mathrm{~b}$ ). We note that the electron density of assynthesized, $\left\langle\mathrm{n}_{\text {sys }}\right\rangle$, larger NCs is more than that for smaller NCs, however, less electron density is added on chemical reduction, $\left\langle\mathrm{n}_{\text {titr }}\right\rangle$, as compared to small sized NCs. This offset between $\left\langle\mathrm{n}_{\text {sys }}\right\rangle$ and $\left\langle\mathrm{n}_{\text {titr }}\right\rangle$ leads to stagnancy in the $\left\langle\mathrm{n}_{\max }\right\rangle$. The constancy of $\left\langle\mathrm{n}_{\max }\right\rangle$ at saturation suggests that for the same doping percentage, irrespective of the NC diameter, the Fermi level is at the same energy with respect to the conduction band minimum of $\operatorname{In}_{2} \mathrm{O}_{3}$ at complete chemical reduction. These observations are in excellent correlation with those made during both the chemical reduction and photodoping of undoped $\mathrm{ZnO} N C s .{ }^{33,47}$ However, $\left\langle\mathrm{n}_{\max }>\right.$ in undoped $\mathrm{ZnO}$ NCs was found to be on the order of $10^{20} \mathrm{~cm}^{-3}$, which is an order of magnitude smaller than that for doped $\operatorname{In}_{2} \mathrm{O}_{3}$ NCs, where aliovalent doping is expected to increase the electron density. ${ }^{13,17,45}$ Very similar trends in electron density are obtained with size series of $\mathrm{Sn}: \mathrm{In}_{2} \mathrm{O}_{3}$ NCs with different $\mathrm{Sn}$ at\% (Figure S17).
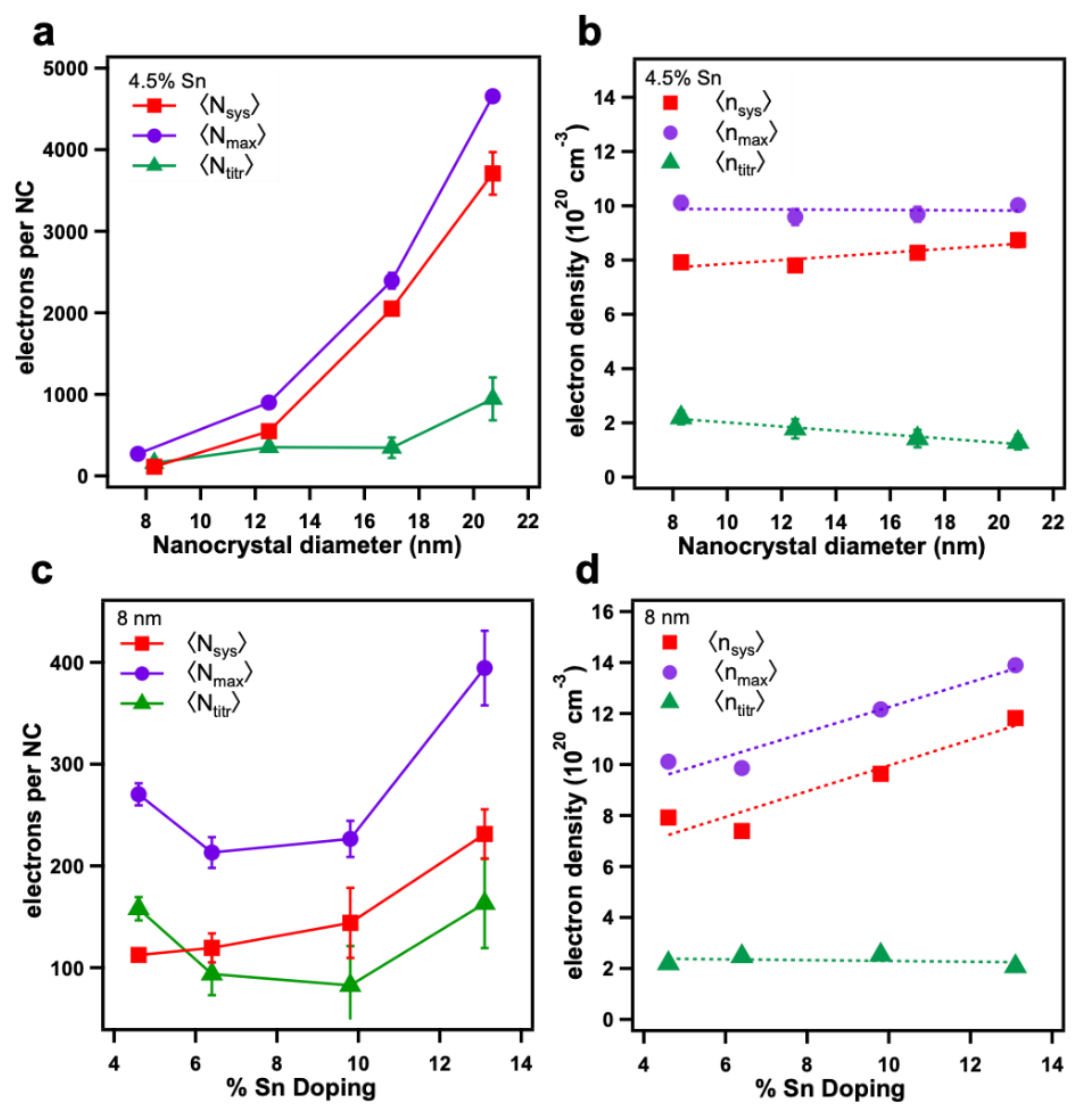

Figure 6: Variation of (a) electrons per NC (b) electron density, as a function of nanocrystal diameter in $4.5 \% \mathrm{Sn}: \mathrm{In}_{2} \mathrm{O}_{3} \mathrm{NCs}$. Variation of (c) electron per $\mathrm{NC}$ (d) electron density, as a function of Sn doping concentration in $8 \mathrm{~nm}$ NCs. Symbols are data points; solid lines are a guide to the eye and dashed lines are least square fit to the data points.

Analogous to the results of the size series, for the doping series, volumetric addition of $\mathrm{CoCp}^{*} 2$ leads to an increase in the electron density and simultaneous increase in the volume fraction of the plasmonic core (Figure S18). The maximum number of electrons per NC is quite similar for 
different doping percentages (Figure 6c, S16). Moreover, the electron density of the as-synthesized $\mathrm{NCs},\left\langle\mathrm{n}_{\mathrm{sys}}\right\rangle$, increases in an approximately linear fashion with $\mathrm{Sn}$ doping percentage (Figure $6 \mathrm{~d}$, S19), indicating a near-constant fraction of activated dopants. ${ }^{28}$ The electron density introduced through the chemical reduction, $\left\langle\mathrm{n}_{\text {titr }}\right\rangle$, is insensitive to the Sn doping concentration (Figure $6 \mathrm{~d}$ ) and is $\sim 2.38 \times 10^{20} \mathrm{~cm}^{-3}$ which causes $\left\langle\mathrm{n}_{\max }\right\rangle$ to increase with doping in the same way as $\left\langle\mathrm{n}_{\text {sys }}\right\rangle$. Photodoping studies on a doping series of $\mathrm{Sn}: \mathrm{In}_{2} \mathrm{O}_{3} \mathrm{NCs}$ and other doped metal oxides have reported similar observations. ${ }^{17,32-33,35}{ }^{45}$ Here, we confirm and interpret these trends by analyzing the distribution of electrons between the core and depletion layer as chemical doping proceeds.

For a given doping percentage, larger NCs contain a larger number of electrons $\left(\left\langle\mathrm{N}_{\mathrm{sys}}\right\rangle\right)$ and a higher electron density $\left(\left\langle\mathrm{n}_{\mathrm{sys}}\right\rangle\right)$ after the synthesis, as compared to smaller NCs. Since the doping percentage is the same for all NCs in a size series, the width of the depletion layer is close to constant but constitutes a different volume fraction for each NC depending upon the diameter of the NC. When electrons are added through chemical doping, larger NCs can accommodate a larger number of electrons per $\mathrm{NC}\left(\left\langle\mathrm{N}_{\mathrm{titr}}\right\rangle\right)$ in their relatively large depletion volume. However, the electron concentration change in the core $\left(\left\langle\mathrm{n}_{\text {titr }}\right\rangle\right)$ is lower. Thus, with an increase in the diameter of the NC, $\left\langle\mathrm{N}_{\text {titr }}\right\rangle$ increases but $\left(\left\langle\mathrm{n}_{\text {titr }}\right\rangle\right)$ decreases (Figure 6a,b). Since this trend runs opposite to the trend for $\left(\left\langle\mathrm{n}_{\text {sys }}\right\rangle\right)$, the maximum electron density after chemical doping, $\left(\left\langle\mathrm{n}_{\max }\right\rangle=\left\langle\mathrm{n}_{\text {sys }}\right\rangle+\right.$ $\left.\left\langle\mathrm{n}_{\text {titr }}\right\rangle\right)$ is approximately constant with size. Further, since smaller NCs having a smaller $\left\langle\mathrm{n}_{\text {sys }}\right\rangle$ add a correspondingly larger $\left\langle\mathrm{n}_{\text {titr }}\right\rangle$, there is a larger change in total electron density, which leads to larger LSPR shift (equation 1) for smaller NCs than larger NCs having the same Sn doping percentage.

For a fixed NC diameter, NCs with a higher doping percentage logically contain a larger number of electrons per $\mathrm{NC}\left(\left\langle\mathrm{N}_{\mathrm{sys}}\right\rangle\right)$ and a higher electron density $\left(\left\langle\mathrm{n}_{\text {sys }}\right\rangle\right)$ after the synthesis than $\mathrm{NCs}$ with smaller doping percentage (Figure $6 \mathrm{c}, \mathrm{d}$ ). Since the width of the depletion region is dependent upon the doping percentage, NCs with a higher doping percentage generally have a larger plasmonic core than NCs with a smaller doping percentage. Considering that we have employed relatively large doping percentages throughout our study $(>4.5 \% \mathrm{Sn})$, the volume fractions of our plasmonic cores are high and do not vary drastically for different doping percentages (Figure S18). ${ }^{38}$ Nonetheless, when electrons are supplied through chemical doping, the size of the plasmonic core increases along with a concomitant increase in the core electron density for all NCs (Figure S18). Irrespective of the doping percentage, there is an almost constant addition of $\left\langle\mathrm{n}_{\text {titr }}\right\rangle$ $\sim 2.38 \times 10^{20} \mathrm{~cm}^{-3}$ to the total electron density for different doping percentages of $8 \mathrm{~nm} \mathrm{Sn}: \operatorname{In}_{2} \mathrm{O}_{3}$ NCs (Figure 6d). We note that this is not the maximum achievable electron density in the NCs, as the volume fraction of the plasmonic core hasn't reached unity for most samples (Figure 5c and S13). If no depletion were present, the maximum electron density in the NC may have been even higher than what was observed. Considering that the number of $\mathrm{CoCp}_{2}{ }_{2}$ molecules is much larger than the number of NCs present in the solution (Figure S6), the data suggest that there is a thermodynamic limit to the maximum electron density that can be transferred from $\mathrm{CoCp} *_{2}$ to the $\mathrm{NC}$. Electron transfer between $\mathrm{CoCp}^{*}{ }_{2}$ and the NCs happens due to a difference in the chemical potential of both the species. As electrons are added to the NCs, the chemical potential (Fermi level) of the electrons increases, thereby decreasing the mismatch in chemical potential. It is possible that once a certain electron density has been transferred to $\mathrm{NC}$, the chemical potential of 
the reducing agent equilibrates with the NC Fermi level and no additional electrons can be transferred to the NC conduction band. An increase in the Sn doping percentage increases the electron density by lowering down the energy of the conduction band minimum while the Fermi level stays at the same chemical potential. ${ }^{45}$ Since the absolute position of the Fermi level is not affected by the doping percentage, this thermodynamic limit is independent of the Sn doping percentage. ${ }^{17,45}$ This limit is also evident from the observation that when the chemical potential of the electrons is raised by employing larger NCs having the same doping percentage, the maximum electron density that can be transferred to the NCs decreased further to $1.4 \times 10^{20} \mathrm{~cm}^{-3}$ for $21 \mathrm{~nm}$ (Figure S19). Previous reports also suggests that when a reducing agent with lower chemical potential was employed, a smaller electron density is transferred from the reducing agent to the NCs. ${ }^{33,35}$ Nonetheless, although the same $\sim 2.38 \times 10^{20} \mathrm{~cm}^{-3}$ electron density is added to all NCs in a doping series of $8 \mathrm{~nm} \mathrm{Sn}: \mathrm{In}_{2} \mathrm{O}_{3} \mathrm{NCs}$, there is a larger relative change in the electron density of NCs with lower as-synthesized doping concentrations than high as-synthesized doping concentration. This increase in electron density leads to the increased LSPR frequency modulation capable at lower doping percentages (see equation 1). Once the maximum electron density $\left\langle\mathrm{n}_{\max }\right\rangle$ is reached and no more electron density can be transferred to the Fermi level of NCs, the electron density is likely being transferred to other chemical species such as surface ligands which leads to a loss of colloidal stability of NCs.

In conclusion, chemical doping allows a clear elucidation of the effects of surface depletion on the dynamic optical properties of doped metal oxide NCs, without convoluting effects of LSPR coupling. Volumetric additions of the reducing agent CoCp* ${ }_{2}$ to colloidal dispersions of $\operatorname{Sn}: \operatorname{In}_{2} \mathrm{O}_{3}$ NCs systematically shift the LSPR energy and increase the extinction. The maximum shift in the LSPR was found to depend on NC diameter and Sn doping concentration, qualitatively consistent with reports on electrochemical charging, ${ }^{19}, 30$ but quantitatively the maximum shift was significantly larger for chemical doping. By fitting the LSPR extinction data with a recently developed Drude model that accounts for heterogeneity in doping and NC diameter and the effects of surface depletion, ${ }^{38}$ we extracted the electron densities and undepleted core volume fractions during the chemical titration and found that the increase in LSPR energy was accompanied by a simultaneous increase in both quantities. We established that the maximum electron density achievable through chemical titration is independent of the NC diameter and depends only on the doping percentage, thus generalizing the conclusions of previous reports on photodoping of a single size of $\mathrm{Sn}: \mathrm{In}_{2} \mathrm{O}_{3} \mathrm{NCs}$ that were analyzed by electron counting titrations. Larger (smaller) NCs have a higher (lower) as-synthesized electron density, so they can accommodate less (more) additional electron density through chemical doping leading to an almost size-independent maximum electron density. We propose that an equilibration of the chemical potential of NCs with the reducing agents restricts the additional transfer of electrons beyond a dopant concentrationdependent maximum electron concentration. These results provide a systematic understanding of post-synthetic modulation of LSPR as it occurs in individual doped metal oxide NCs and lays an important foundation for interpreting modulation in thin films, like those investigated for smart window applications. ${ }^{7}$ Similar investigations using chemical titrations to interrogate other doped metal oxide NC compositions will shed light on the relevance of surface depletion in understanding properties across this emerging class of plasmonic nanocrystals, with implications for their potential applications in catalysis, sensing, therapeutics, and more. $5,10,26,29,48$ 


\section{Supporting Information}

Details of nanocrystal synthesis and characterization (STEM), chemical titration data of samples not shown in the main text and HEDA fits along with parameters obtained are provided in the supporting information and can be accessed free of charge from the ACS Publications website.

\section{Author Information}

$\dagger$ B. Tandon and S. A. Shubert-Zuleta contributed equally to the manuscript.

*E-mail: milliron@che.utexas.edu.

The authors declare no competing financial interest.

\section{Acknowledgements}

The authors acknowledge support from the National Science Foundation (NSF) including CHE1905263 and a Graduate Research Fellowship under award number DGE-1610403 and DGE2137420. Additional support was provided by the Welch Foundation (F-1848).

\section{Experimental Section:}

Chemicals: For the synthesis and characterization of Sn-doped $\operatorname{In}_{2} \mathrm{O}_{3}\left(\mathrm{Sn}_{\mathrm{In}_{2}} \mathrm{O}_{3}\right)$ nanocrystals (NCs), all chemicals were acquired commercially and employed without further purification prior to their use. Indium(III) acetate (STREM, $\geq 99.99 \%$ ), tin(IV) acetate (Sigma-Aldrich 99.99\%), oleyl alcohol (Sigma-Aldrich, 85\%) and oleic acid (Sigma-Aldrich, 90\%) were used for the synthesis of different Sn-doped $\mathrm{In}_{2} \mathrm{O}_{3}$ NCs. Oleylamine (Sigma-Aldrich, 70\%), ethanol (Fischer Chemical, 90\%), hexane (Fischer Chemical $\geq 99.9 \%$ ), tetrahydrofuran (Sigma-Aldrich, $\geq 99.9 \%$ ) and toluene (Sigma-Aldrich, 99.8\%) were utilized for washing the resulting nanocrystals and characterizing them through different techniques. For chemical reduction of $\mathrm{Sn}: \mathrm{In}_{2} \mathrm{O}_{3} \mathrm{NCs}_{\text {sith }}$ decamethylcaboltocene, Sodium tetrakis[3,5-bis(trifluoromethyl)phenyl]borate and Bis(pentamethylcyclopentadienyl)cobalt(II) were acquired from Sigma-Aldrich and dried prior to their use.

\section{Nanocrystal Synthesis:}

Sn-doped $\mathrm{In}_{2} \mathrm{O}_{3} \mathrm{NCs}$ of different sizes and doping percentages were synthesized following the slow-injection synthetic procedure developed by the Hutchison group. ${ }^{39-40}$ In brief, on a standard Schlenk line apparatus, In and Sn precursor in a desired stoichiometric ratio were dispersed in oleic acid to yield a $0.5 \mathrm{M}$ solution and this mixture was degassed at $100^{\circ} \mathrm{C}$ under vacuum conditions. This was followed by an undisturbed heating at $150^{\circ} \mathrm{C}$ and constant stirring under $\mathrm{N}_{2}$ atmosphere for 2 hours. Using a syringe pump, different volumes of this mixture were subsequently injected at the rate of $0.35 \mathrm{~mL} / \mathrm{min}$ into different flasks containing $13 \mathrm{~mL}$ oleyl alcohol kept at $290{ }^{\circ} \mathrm{C}$ (under $\mathrm{N}_{2}$ conditions). Injection of different volumes yielded NCs with a constant doping percentage but different sizes. Synthesized NCs were separated from the reaction mixture by repeated precipitation with anti-solvent ethanol, centrifugation at $9000 \mathrm{rpm}$ for $5 \mathrm{~min}$, and redispersing in hexane before finally being prepared as a colloidal dispersion of NCs in hexane. 
During each cycle of centrifugation, extra oleic acid and oleylamine were added as ligands to stabilize the NC dispersion.

\section{Nanocrystal Characterization:}

Scanning transmission electron microscopy (STEM): The average NC diameter, polydispersity and morphology of the $\mathrm{Sn}: \mathrm{In}_{2} \mathrm{O}_{3} \mathrm{NCs}$ were examined through low-resolution STEM microscopy on a Hitachi S5500 operating at an accelerating voltage of $30 \mathrm{mV}$ in the STEM mode. Sample were prepared by drop-casting a $20 \mu \mathrm{L}$ dilute solution of NCs in hexane $(\sim 15 \mathrm{mg} / \mathrm{mL}$ of NCs in 1 $\mathrm{mL}$ hexane) on a copper 300 mesh TEM grid and drying it in vacuum overnight. The average NC diameter and standard deviation in sizes of different NCs were determined by analyzing 200-300 particles from their respective STEM images using ImageJ software and fitting the statistics to a Gaussian size distribution.

Inductively coupled plasma-atomic emission spectroscopy (ICP-AES): Sn doping percentages in $\mathrm{In}_{2} \mathrm{O}_{3}$ and volume fraction of NCs in solution was experimentally determined by utilizing ICPAES technique on a Varian 720-ES ICP Optical Emission Spectrometer. Samples for the ICP-AES were processed by digesting a known volume of the stock solution of $\mathrm{NC}$ in aqua-regia mixture for 48 hours. This was followed by diluting the aqua-regia solution with milli-Q water such that the total acid concentration becomes approximately $2 \% \mathrm{v} / \mathrm{v}$. Standard solutions of variable concentration for different elements were prepared by diluting the commercial ICP-AES standard with $2 \% \mathrm{HNO}_{3}$ solution in milli-Q water. The $\mathrm{Sn}$ doping percentage was calculated by taking a mole fraction of Sn concentration with respect to the total metal concentration $(\mathrm{In}+\mathrm{Sn})$ obtained in the ICP-AES. The volume fraction of $\mathrm{Sn}: \mathrm{In}_{2} \mathrm{O}_{3} \mathrm{NCs}$ was calculated from the concentration of In and $\mathrm{Sn}$ in the analyte using an assumed stoichiometry of $(\mathrm{In}+\mathrm{Sn})_{2} \mathrm{O}_{3}$ and density of $7140 \mathrm{mg} / \mathrm{mL}$.

\section{Chemical reduction of $\mathrm{Sn}: \mathrm{In}_{2} \mathrm{O}_{3} \mathrm{NCs}$ with decamethylcobaltocene $\left(\mathrm{CoCp}_{2}{ }_{2}\right)$ :}

For the chemical reduction of $\mathrm{Sn}: \mathrm{In}_{2} \mathrm{O}_{3} \mathrm{NCs}, 0.02 \mathrm{M} \mathrm{CoCp} * 2$ solution in tetrahydrofuran (THF):toluene (1:1) mixture and $0.02 \mathrm{M}\left[\mathrm{H}\left(\mathrm{OEt}_{2}\right)\right]^{+}\left[\mathrm{BAr}_{4}\right]^{-}$acid solution in THF was first prepared following literature reports. Afterwards, NCs from their hexane solution were first precipitated with ethanol and a stock solution of NCs in anhydrous THF:toluene (1:1) mixture was prepared. To perform the titrations, $50 \mu \mathrm{L}$ of $0.02 \mathrm{M}\left[\mathrm{H}\left(\mathrm{OEt}_{2}\right)\right]^{+}\left[\mathrm{BAr}^{\mathrm{F}}\right]^{-}$solution was added to $200 \mathrm{uL}$ of NC stock solution in a quartz cuvette with $1 \mathrm{~mm}$ pathlength. Subsequently, $5 \mathrm{uL}$ aliquots of $0.02 \mathrm{M} \mathrm{CoCp} *_{2}$ solution were added to this mixture and shaken. During each addition to the quartz cuvette, in situ spectroscopy measurements were performed in absorbance mode with a fiber-coupled ASD Inc. PANalytical spectrometer. To establish equilibrium, each measurement was carried out 1.5 minutes after the addition of an aliquot. For each sample, titrations were repeated three times for the same volume of the stock solution to check reproducibility in data.

\section{Chemical oxidation of $\mathrm{Sn}: \mathrm{In}_{2} \mathrm{O}_{3} \mathrm{NCs}$ with 2,4,6-tri-tert-butylphenoxy radical (t-Bu3ArO॰):}

t-Bu $3 \mathrm{ArO}^{\circ}$ solution was prepared from the parent phenol, 2,4,6-tri-tert-butylphenol, by following earlier literature reports. ${ }^{49}$ The chemical oxidation of $\mathrm{Sn}: \mathrm{In}_{2} \mathrm{O}_{3} \mathrm{NCs}$ was carried out by first reducing the NCs to the LSPR saturation point using $0.02 \mathrm{M} \mathrm{CoCp} *_{2}$ and thereafter, subsequent

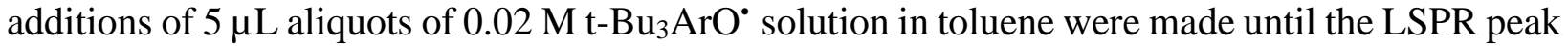
position corresponded to the LSPR energy of the as-synthesized NCs.

\section{Heterogeneous Ensemble Drude Approximation (HEDA) Model:}


A detailed discussion of this analytical model and a copy of the MATLAB script that runs the fitting procedure can be found in reference 38. To summarize, the HEDA model uses four input values obtained from experimental data, volume fraction of NCs in the dispersion $f_{v}$, the average diameter $\mu_{r}$, and the standard deviation in diameter $\sigma_{r}$, along with an experimental extinction spectrum of a dilute solution of NCs. From this, the HEDA model fits for four optoelectronic variables, the average electron concentration $n_{e}$, the standard deviation in a normal distribution of charge electron concentrations $\sigma_{n_{e}}$, the mean free path of a charge electron $l_{M F P}$, and the electron accessible volume fraction $f_{e}$. The model accounts for heterogeneity, unlike the simple Drude approximation, by considering distributions in the size and electron concentration. Additionally, the depletion layer and surface scattering are considered in the optical response to improve upon the Drude approximation. A successful fitting procedure enables the determination of the properties of an average NC in an ensemble, unclouded by the effects of heterogeneity.

In our fitting procedure, it was necessary to place physically realistic bounds on the fit parameters, $l_{M F P}$ and $\sigma_{n_{e}}$. An upper bound of $17 \mathrm{~nm}$ was placed on $l_{M F P}$ because as the NC radius is decreased to become much smaller than the mean free path, surface scattering becomes the dominant damping mechanism and bulk damping becomes negligible. The fit becomes insensitive to the value of $l_{M F P}$, which tends to increase indefinitely if not constrained; constraining $l_{M F P}$ to a maximum $17 \mathrm{~nm}$ circumvents this issue. The lower bound placed on the $\sigma_{n_{e}}$ parameter is attributed to the minimum standard deviation in the number of dopant atoms incorporated into a single NC. This value is approximated by Poissonian statistics to follow the relationship: $\sigma_{N_{d}} \approx \sqrt{\mu_{N_{d}}}$. After quantifying the dopant concentration in each sample by ICP-AES, we set the lower boundary of $\sigma_{N_{d}}$ to $\sqrt{\mu_{N_{d}}}$

\section{References}

1. Willets, K. A.; Duyne, R. P. V., Localized Surface Plasmon Resonance Spectroscopy and Sensing. Ann. Rev. Phys. Chem. 2007, 58, 267-297.

2. Faucheaux, J. A.; Stanton, A. L. D.; Jain, P. K., Plasmon Resonances of Semiconductor Nanocrystals: Physical Principles and New Opportunities. J.Phys. Chem. Lett. 2014, 5, 976-985.

3. Tandon, B.; Ashok, A.; Nag, A., Colloidal Transparent Conducting Oxide Nanocrystals: A New Infrared Plasmonic Material. Pramana 2015, 84, 1087-1098.

4. Agrawal, A.; Cho, S. H.; Zandi, O.; Ghosh, S.; Johns, R. W.; Milliron, D. J., Localized Surface Plasmon Resonance in Semiconductor Nanocrystals. Chem. Rev. 2018, 118, 3121-3207.

5. Jain, P. K.; Huang, X.; El-Sayed, I. H.; El-Sayed, M. A., Noble Metals on the Nanoscale: Optical and Photothermal Properties and Some Applications in Imaging, Sensing, Biology, and Medicine. Acc. Chem. Res. 2008, 41, 1578-1586.

6. Atwater, H. A.; Polman, A., Plasmonics for Improved Photovoltaic Devices. Nat. Mater. 2010, 9, 205-213.

7. Garcia, G.; Buonsanti, R.; Runnerstrom, E. L.; Mendelsberg, R. J.; Llordes, A.; Anders, A.; Richardson, T. J.; Milliron, D. J., Dynamically Modulating the Surface Plasmon Resonance of Doped Semiconductor Nanocrystals. Nano Lett. 2011, 11, 4415-4420.

8. Garcia, G.; Buonsanti, R.; Llordes, A.; Runnerstrom, E. L.; Bergerud, A.; Milliron, D. J., NearInfrared Spectrally Selective Plasmonic Electrochromic Thin Films. Adv. Opt. Mater. 2013, 1, 215-220.

9. Wang, Y.; Runnerstrom, E. L.; Milliron, D. J., Switchable Materials for Smart Windows. Annual Rev. Chem. Biomol. Eng. 2016, 7, 283-304. 
10. Haes, A. J.; Hall, W. P.; Chang, L.; Klein, W. L.; Van Duyne, R. P., A Localized Surface Plasmon Resonance Biosensor: First Steps toward an Assay for Alzheimer's Disease. Nano Lett. 2004, 4, 1029-1034.

11. Luther, J. M.; Jain, P. K.; Ewers, T.; Alivisatos, A. P., Localized Surface Plasmon Resonances Arising from Free Carriers in Doped Quantum Dots. Nat. Mater. 2011, 10, 361-366.

12. Scotognella, F.; Della Valle, G.; Srimath Kandada, A. R.; Dorfs, D.; Zavelani-Rossi, M.; Conforti, M.; Miszta, K.; Comin, A.; Korobchevskaya, K.; Lanzani, G.; Manna, L.; Tassone, F., Plasmon Dynamics in Colloidal $\mathrm{Cu}_{2-x}$ Se Nanocrystals. Nano Lett. 2011, 11, 4711-4717.

13. Lounis, S. D.; Runnerstrom, E. L.; Llordés, A.; Milliron, D. J., Defect Chemistry and Plasmon Physics of Colloidal Metal Oxide Nanocrystals. J. Phys. Chem. Lett. 2014, 5, 1564-1574.

14. Comin, A.; Manna, L., New Materials for Tunable Plasmonic Colloidal Nanocrystals. Chem. Soc. Rev. 2014, 43, 3957-3975.

15. Balitskii, O. A.; Sytnyk, M.; Stangl, J.; Primetzhofer, D.; Groiss, H.; Heiss, W., Tuning the Localized Surface Plasmon Resonance in $\mathrm{C}_{\mathrm{u} 2-\mathrm{x}} \mathrm{Se}$ Nanocrystals by Postsynthetic Ligand Exchange. ACS Appl. Mater. Interfaces 2014, 6, 17770-17775.

16. Kriegel, I.; Jiang, C.; Rodríguez-Fernández, J.; Schaller, R. D.; Talapin, D. V.; da Como, E.; Feldmann, J., Tuning the Excitonic and Plasmonic Properties of Copper Chalcogenide Nanocrystals. J. Am. Chem. Soc. 2012, 134, 1583-1590.

17. Schimpf, A. M.; Lounis, S. D.; Runnerstrom, E. L.; Milliron, D. J.; Gamelin, D. R., Redox Chemistries and Plasmon Energies of Photodoped $\mathrm{In}_{2} \mathrm{O}_{3}$ and $\mathrm{Sn}$-Doped $\mathrm{In}_{2} \mathrm{O}_{3}$ (ITO) Nanocrystals. J. Am. Chem. Soc. 2015, 137, 518-524.

18. Schimpf, A. M.; Knowles, K. E.; Carroll, G. M.; Gamelin, D. R., Electronic Doping and RedoxPotential Tuning in Colloidal Semiconductor Nanocrystals. Acc. Chem. Res. 2015, 48, 1929-1937. 19. Tandon, B.; Agrawal, A.; Heo, S.; Milliron, D. J., Competition between Depletion Effects and Coupling in the Plasmon Modulation of Doped Metal Oxide Nanocrystals. Nano Lett. 2019, 19, 2012-2019.

20. Kanehara, M.; Koike, H.; Yoshinaga, T.; Teranishi, T., Indium Tin Oxide Nanoparticles with Compositionally Tunable Surface Plasmon Resonance Frequencies in the Near-IR Region. J. Am. Chem. Soc. 2009, 131, 17736-17737.

21. Runnerstrom, E. L.; Bergerud, A.; Agrawal, A.; Johns, R. W.; Dahlman, C. J.; Singh, A.; Selbach, S. M.; Milliron, D. J., Defect Engineering in Plasmonic Metal Oxide Nanocrystals. Nano Lett. 2016, 16, 3390-3398.

22. Fang, H.; Hegde, M.; Yin, P.; Radovanovic, P. V., Tuning Plasmon Resonance of $\operatorname{In}_{2} \mathrm{O}_{3}$ Nanocrystals throughout the Mid-Infrared Region by Competition between Electron Activation and Trapping. Chem. Mater. 2017, 29, 4970-4979.

23. Tandon, B.; Ghosh, S.; Milliron, D. J., Dopant Selection Strategy for High-Quality Factor Localized Surface Plasmon Resonance from Doped Metal Oxide Nanocrystals. Chem. Mater. 2019, 31, 7752-7760.

24. Tandon, B.; Gibbs, S. L.; Zydlewski, B. Z.; Milliron, D. J., Quantitative Analysis of Plasmonic Metal Oxide Nanocrystal Ensembles Reveals the Influence of Dopant Selection on Intrinsic Optoelectronic Properties. Chem. Mater. 2021, 33, 6955-6964.

25. Gibbs, S. L.; Staller, C. M.; Milliron, D. J., Surface Depletion Layers in Plasmonic Metal Oxide Nanocrystals. Acc. Chem. Res. 2019, 52, 2516-2524.

26. Weiss, E. A., Designing the Surfaces of Semiconductor Quantum Dots for Colloidal Photocatalysis. ACS Energy Lett. 2017, 2, 1005-1013. 
27. Staller, C. M.; Robinson, Z. L.; Agrawal, A.; Gibbs, S. L.; Greenberg, B. L.; Lounis, S. D.; Kortshagen, U. R.; Milliron, D. J., Tuning Nanocrystal Surface Depletion by Controlling Dopant Distribution as a Route Toward Enhanced Film Conductivity. Nano Lett. 2018, 18, 2870-2878.

28. Staller, C. M.; Gibbs, S. L.; Saez Cabezas, C. A.; Milliron, D. J., Quantitative Analysis of Extinction Coefficients of Tin-Doped Indium Oxide Nanocrystal Ensembles. Nano Lett. 2019, 19, 8149-8154.

29. Jhong, H.-R. M.; Nwabara, U. O.; Shubert-Zuleta, S.; Grundish, N. S.; Tandon, B.; Reimnitz, L. C.; Staller, C. M.; Ong, G. K.; Saez Cabezas, C. A.; Goodenough, J. B.; Kenis, P. J. A.; Milliron, D. J., Efficient Aqueous Electroreduction of $\mathrm{CO}_{2}$ to Formate at Low Overpotential on Indium Tin Oxide Nanocrystals. Chem. Mater. 2021 doi.org/10.1021/acs.chemmater.1c01649.

30. Zandi, O.; Agrawal, A.; Shearer, A. B.; Reimnitz, L. C.; Dahlman, C. J.; Staller, C. M.; Milliron, D. J., Impacts of Surface Depletion on the Plasmonic Properties of Doped Semiconductor Nanocrystals. Nat. Mater. 2018, 17, 710-717.

31. Agrawal, A.; Kriegel, I.; Runnerstrom, E. L.; Scotognella, F.; Llordes, A.; Milliron, D. J., Rationalizing the Impact of Surface Depletion on Electrochemical Modulation of Plasmon Resonance Absorption in Metal Oxide Nanocrystals. ACS Photonics 2018, 5, 2044-2050.

32. Valdez, C. N.; Braten, M.; Soria, A.; Gamelin, D. R.; Mayer, J. M., Effect of Protons on the Redox Chemistry of Colloidal Zinc Oxide Nanocrystals. J. Am. Chem. Soc. 2013, 135, 8492-8495. 33. Valdez, C. N.; Schimpf, A. M.; Gamelin, D. R.; Mayer, J. M., Proton-Controlled Reduction of ZnO Nanocrystals: Effects of Molecular Reductants, Cations, and Thermodynamic Limitations. $J$. Am. Chem. Soc. 2016, 138, 1377-1385.

34. Ghosh, S.; Castillo-Lora, J.; Soudackov, A. V.; Mayer, J. M.; Hammes-Schiffer, S., Theoretical Insights into Proton-Coupled Electron Transfer from a Photoreduced ZnO Nanocrystal to an Organic Radical. Nano Lett. 2017, 17, 5762-5767.

35. Castillo-Lora, J.; Mitsuhashi, R.; Mayer, J. M., Revealing the Relative Electronic Landscape of Colloidal $\mathrm{ZnO}$ and $\mathrm{TiO}_{2}$ Nanoparticles via Equilibration Studies. J. Phys. Chem. C 2019, 123, 10262-10271.

36. Castillo-Lora, J.; Delley, M. F.; Laga, S. M.; Mayer, J. M., Two-Electron-Two-Proton Transfer from Colloidal $\mathrm{ZnO}$ and $\mathrm{TiO}_{2}$ Nanoparticles to Molecular Substrates. J. Phys. Chem. Lett. 2020, $11,7687-7691$.

37. Xu, Y.; Schoonen, M. A. A., The Absolute Energy Positions of Conduction and Valence Bands of Selected Semiconducting Mineral Am. Min. 2000, 85, 543-556.

38. Gibbs, S. L.; Staller, C. M.; Agrawal, A.; Johns, R. W.; Saez Cabezas, C. A.; Milliron, D. J., Intrinsic Optical and Electronic Properties from Quantitative Analysis of Plasmonic Semiconductor Nanocrystal Ensemble Optical Extinction. J. Phys. Chem. C 2020, 124, 2435124360.

39. Jansons, A. W.; Hutchison, J. E., Continuous Growth of Metal Oxide Nanocrystals: Enhanced Control of Nanocrystal Size and Radial Dopant Distribution. ACS Nano 2016, 10, 6942-6951.

40. Jansons, A. W.; Plummer, L. K.; Hutchison, J. E., Living Nanocrystals. Chem. Mater. 2017, 29, 5415-5425.

41. Lounis, S. D.; Runnerstrom, E. L.; Bergerud, A.; Nordlund, D.; Milliron, D. J., Influence of Dopant Distribution on the Plasmonic Properties of Indium Tin Oxide Nanocrystals. J. Am. Chem. Soc. 2014, 136, 7110-7116.

42. Tandon, B.; Yadav, A.; Khurana, D.; Reddy, P.; Santra, P. K.; Nag, A., Size-Induced Enhancement of Carrier Density, LSPR Quality Factor, and Carrier Mobility in Cr-Sn Doped $\mathrm{In}_{2} \mathrm{O}_{3}$ Nanocrystals. Chem. Mater. 2017, 29, 9360-9368. 
43. Brookhart, M.; Grant, B.; Volpe, A. F., [(3,5-( $\left.\left.\left(\mathrm{CF}_{3}\right)_{2} \mathrm{C}_{6} \mathrm{H}_{3}\right)_{4} \mathrm{~B}\right]-\left[\mathrm{H}\left(\mathrm{OEt}_{2}\right)_{2}\right]^{+}$: A Convenient Reagent for Generation and Stabilization of Cationic, Highly Electrophilic Organometallic Complexes. Organometallics 1992, 11, 3920-3922.

44. Connelly, N. G.; Geiger, W. E., Chemical Redox Agents for Organometallic Chemistry. Chem. Rev. 1996, 96, 877-910.

45. Araujo, J. J.; Brozek, C. K.; Liu, H.; Merkulova, A.; Li, X.; Gamelin, D. R., Tunable BandEdge Potentials and Charge Storage in Colloidal Tin-Doped Indium Oxide (ITO) Nanocrystals. ACS Nano 2021, 15, 14116-14124.

46. Liu, Z.; Beaulac, R., Nature of the Infrared Transition of Colloidal Indium Nitride Nanocrystals: Nonparabolicity Effects on the Plasmonic Behavior of Doped Semiconductor Nanomaterials. Chem. Mater. 2017, 29, 7507-7514.

47. Schimpf, A. M.; Gunthardt, C. E.; Rinehart, J. D.; Mayer, J. M.; Gamelin, D. R., Controlling Carrier Densities in Photochemically Reduced Colloidal ZnO Nanocrystals: Size Dependence and Role of the Hole Quencher. J. Am. Chem. Soc. 2013, 135, 16569-16577.

48. Sannomiya, T.; Hafner, C.; Voros, J., in-situ Sensing of Single Binding Events by Localized Surface Plasmon Resonance. Nano Lett. 2008, 8, 3450-3455.

49. Manner, V. W.; Markle, T. F.; Freudenthal, J. H.; Roth, J. P.; Mayer, J. M., The First Crystal Structure of a Monomeric Phenoxyl Radical: 2,4,6-tri-tert-butylphenoxyl Radical. Chem. Commun. 2008, 2, 256-258. 
Investigating the Role of Surface Depletion in Governing Electron Transfer Events in

\title{
Colloidal Plasmonic Nanocrystals
}

\author{
Bharat Tandon $^{\llbracket \dagger}$, Sofia A. Shubert-Zuleta ${ }^{\dagger *}$, Delia J. Milliron ${ }^{\mathbb{I} \neq *}$ \\ ${ }^{I}$ McKetta Department of Chemical Engineering, University of Texas at Austin, \\ Austin, Texas 78712-1589, United States \\ ${ }^{7}$ Department of Chemistry, University of Texas at Austin, Austin, Texas 78712-1589, United \\ States
}

Supporting Information 


\section{Table of Contents:}

Figure S1: STEM images of 6\% Sn: $\operatorname{In}_{2} \mathrm{O}_{3}$ NCs of different diameters ......................

Figure S2: STEM images of $10 \% \mathrm{Sn}: \mathrm{In}_{2} \mathrm{O}_{3} \mathrm{NCs}$ of different diameters $\ldots \ldots \ldots \ldots \ldots \ldots \ldots \ldots \ldots$

Figure S3: STEM images of $13 \% \mathrm{Sn}: \mathrm{In}_{2} \mathrm{O}_{3} \mathrm{NCs}$ of different diameters.......................

Figure S4: Normalized LSPR spectra of size and doping series of $\mathrm{Sn}: \mathrm{In}_{2} \mathrm{O}_{3} \mathrm{NCs} \ldots \ldots \ldots \ldots \ldots . . \ldots$

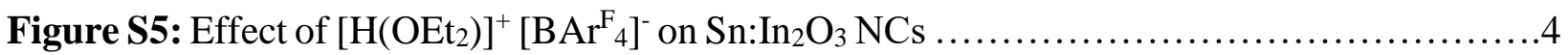

Figure S6: Effect of concentration on LSPR modulation..................................5

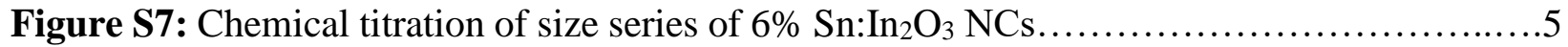

Figure S8: Chemical titration of size series of $10 \% \mathrm{Sn}: \mathrm{In}_{2} \mathrm{O}_{3} \mathrm{NCs} \ldots \ldots \ldots \ldots \ldots \ldots \ldots \ldots \ldots \ldots \ldots$

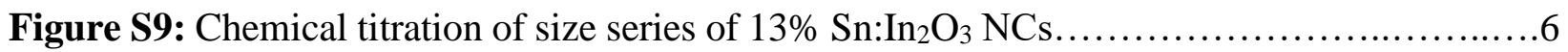

Figure S10: Chemical titration of doping series of $13 \mathrm{~nm} \mathrm{Sn}: \mathrm{In}_{2} \mathrm{O}_{3} \mathrm{NCs} \ldots \ldots \ldots \ldots \ldots \ldots \ldots \ldots . \ldots \ldots$

Figure S11: Chemical titration of doping series of $21 \mathrm{~nm} \mathrm{Sn: \operatorname {In } _ { 2 } \mathrm { O } _ { 3 } \mathrm { NCs } \ldots \ldots \ldots \ldots \ldots \ldots \ldots \ldots . . .}$

Figure S12: HEDA fits to the extinction curves of size and doping series of $\mathrm{Sn}: \mathrm{In}_{2} \mathrm{O}_{3} \mathrm{NCs} \ldots \ldots . .9$

Figure S13: Change in electron density during chemical titration for size and doping series of

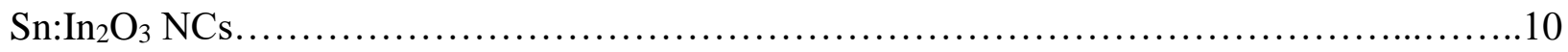

Figure S14: Change in standard deviation of electron denisty during chemical titration of $13 \mathrm{~nm}$, $4.5 \% \mathrm{Sn}: \mathrm{In}_{2} \mathrm{O}_{3} \mathrm{NCs}$

Figure S15: Change in electrons per NC during chemical titrations of size and doping series of

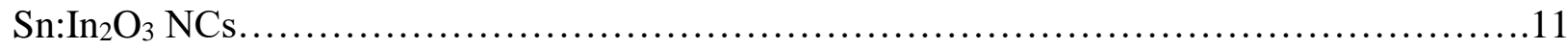

Figure S16: Change in maximum electrons per NC for size and doping series of $\mathrm{Sn}: \operatorname{In}_{2} \mathrm{O}_{3}$ NCs............................................................................ 12

Figure S17: Change in maximum electron density for size series of $\mathrm{Sn}: \mathrm{In}_{2} \mathrm{O}_{3} \mathrm{NCs} \ldots \ldots \ldots \ldots .12$

Figure S18: Change in electron density and undepleted core volume fraction during chemical

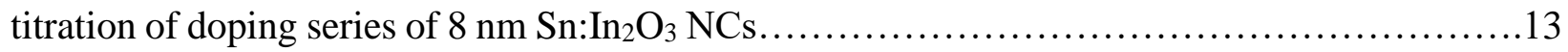

Figure S19: Change in maximum electron density for doping series of $\mathrm{Sn}: \mathrm{In}_{2} \mathrm{O}_{3} \mathrm{NCs} \ldots \ldots \ldots \ldots 13$ 

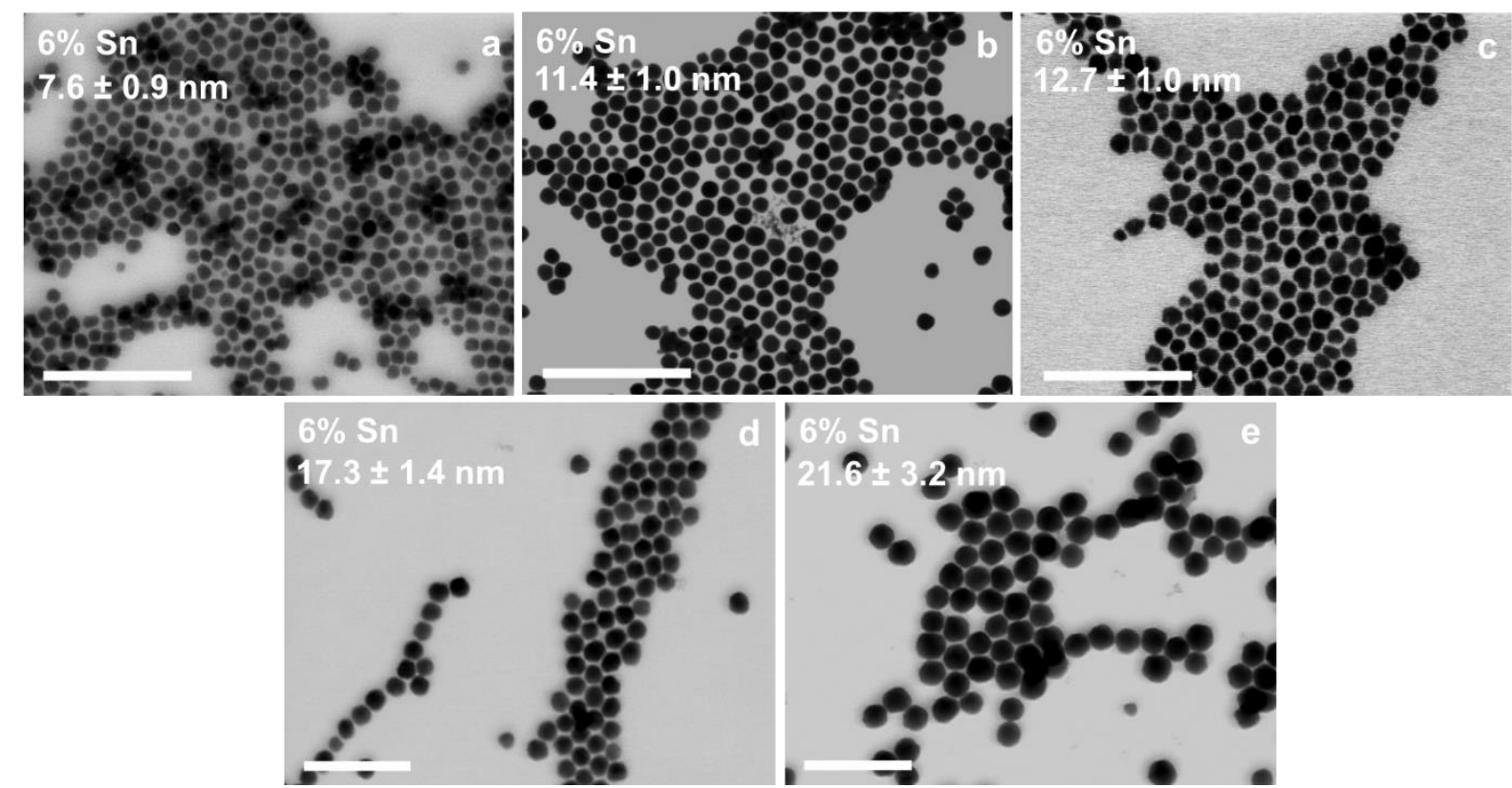

Figure S1: STEM images for different diameters of $6 \% \mathrm{Sn}: \mathrm{In}_{2} \mathrm{O}_{3} \mathrm{NCs}$. All scale bars correspond to $100 \mathrm{~nm}$.
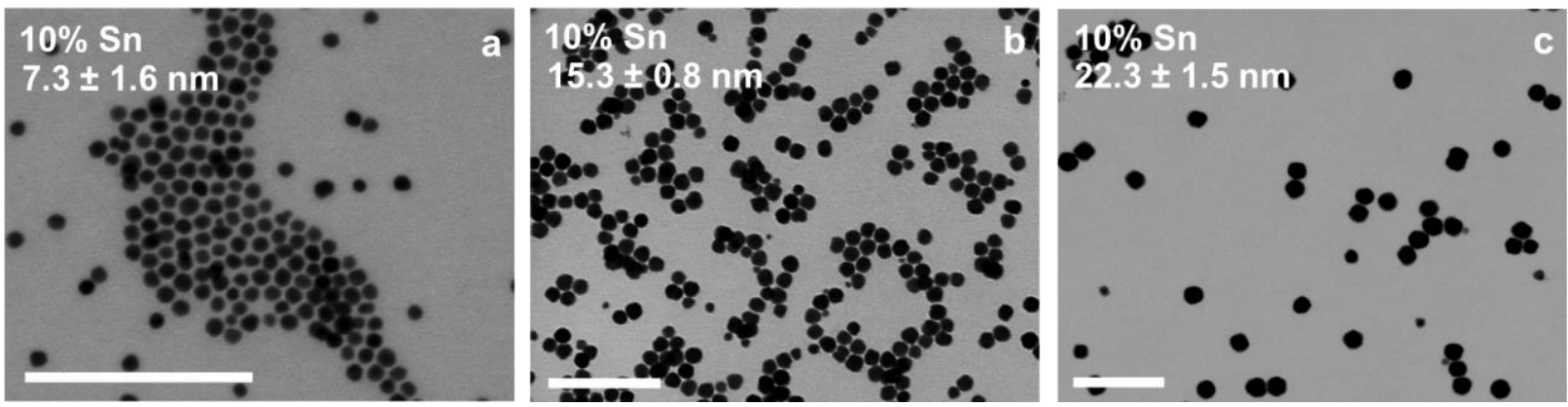

Figure S2: STEM images for different diameters of $10 \% \mathrm{Sn}: \mathrm{In}_{2} \mathrm{O}_{3} \mathrm{NCs}$. All scale bars correspond to $100 \mathrm{~nm}$.
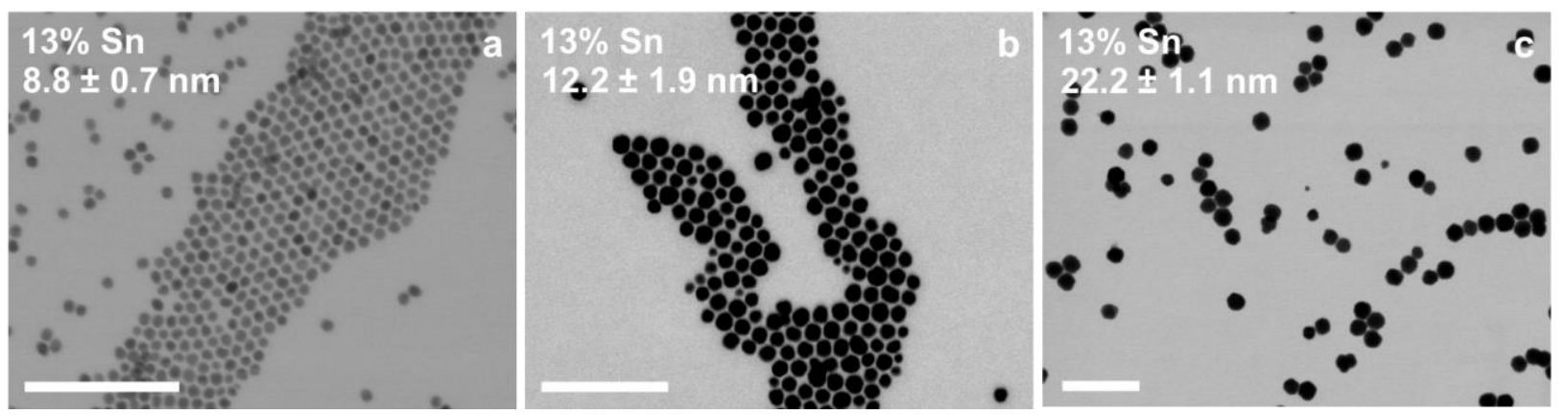

Figure S3: STEM images for different diameters of $13 \% \mathrm{Sn}: \mathrm{In}_{2} \mathrm{O}_{3}$ NCs. All scale bars correspond to $100 \mathrm{~nm}$. 

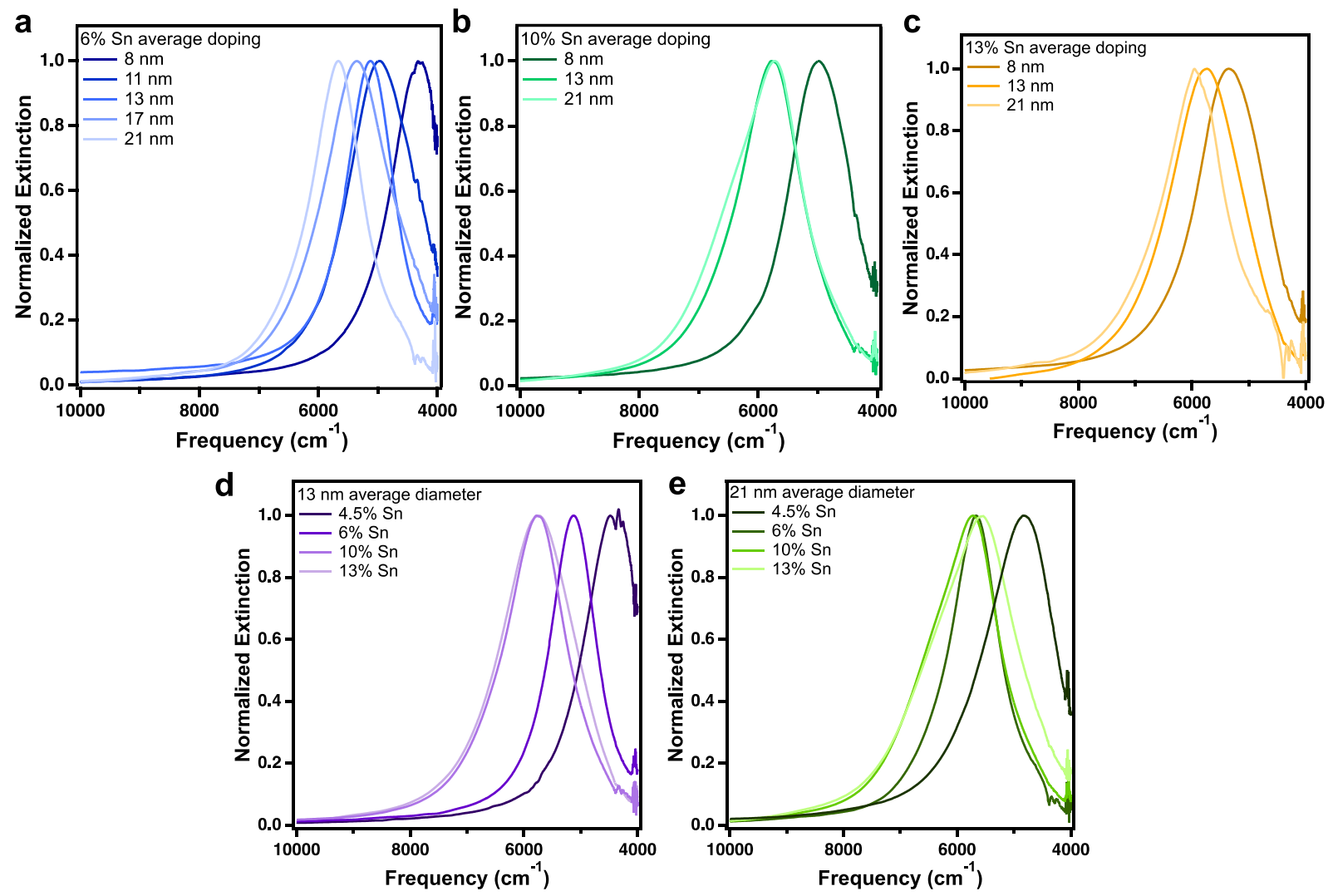

Figure S4: Normalized extinction spectra for (a) $6 \% \mathrm{Sn}: \mathrm{In}_{2} \mathrm{O}_{3} \mathrm{NCs}$ (b) $10 \% \mathrm{Sn}: \mathrm{In}_{2} \mathrm{O}_{3} \mathrm{NCs}$ (c) $13 \% \mathrm{Sn}: \mathrm{In}_{2} \mathrm{O}_{3}$ NCs with different diameters; (d) different $\mathrm{Sn}: \mathrm{In}_{2} \mathrm{O}_{3}$ NCs with $13 \mathrm{~nm}$ as average diameter (e) different $\mathrm{Sn}: \mathrm{In}_{2} \mathrm{O}_{3} \mathrm{NCs}$ with $21 \mathrm{~nm}$ as average diameter.

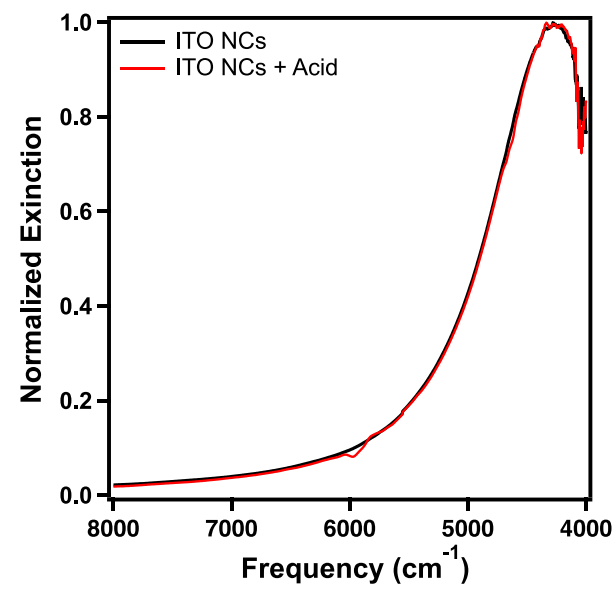

Figure S5: Normalized extinction spectra of $\mathrm{Sn}: \mathrm{In}_{2} \mathrm{O}_{3} \mathrm{NCs}$ before and after addition of $\left[\mathrm{H}\left(\mathrm{OEt}_{2}\right)\right]^{+}$ $\left[\mathrm{BAr}^{\mathrm{F}}\right]^{-}$acid. 

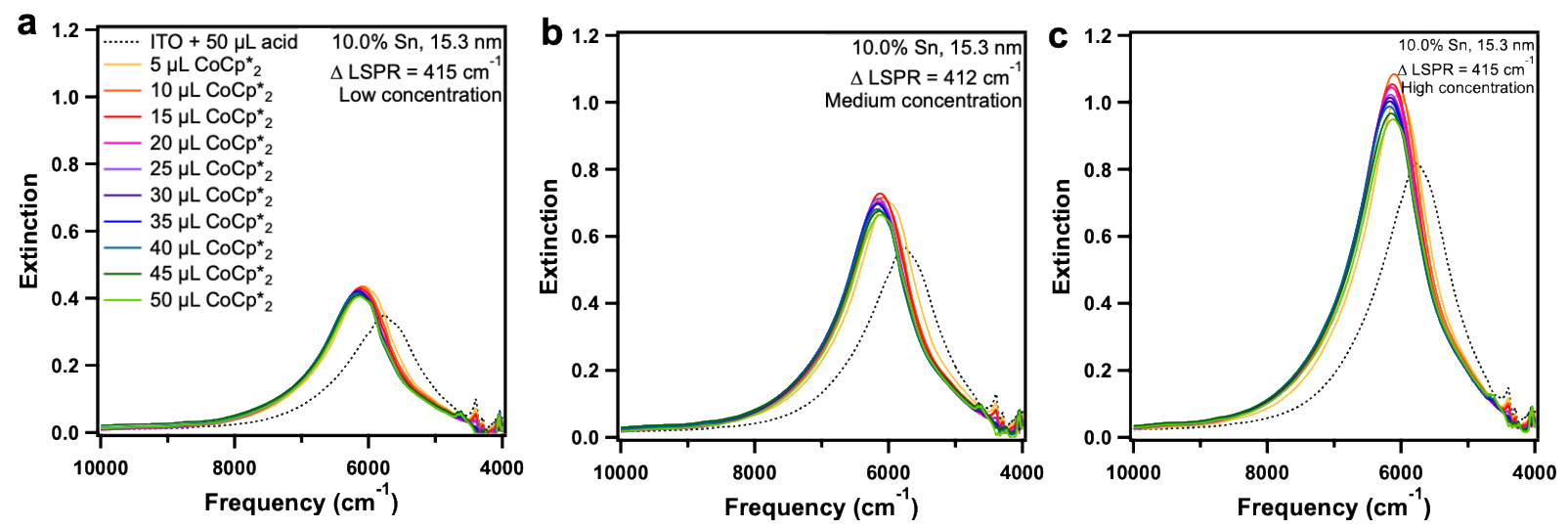

Figure S6: Establishing independence of maximum LSPR shift from the NC concentration by performing chemical reduction on dispersions of $15 \mathrm{~nm} 10 \% \mathrm{Sn}: \mathrm{In}_{2} \mathrm{O}_{3} \mathrm{NCs}$ with different concentrations.
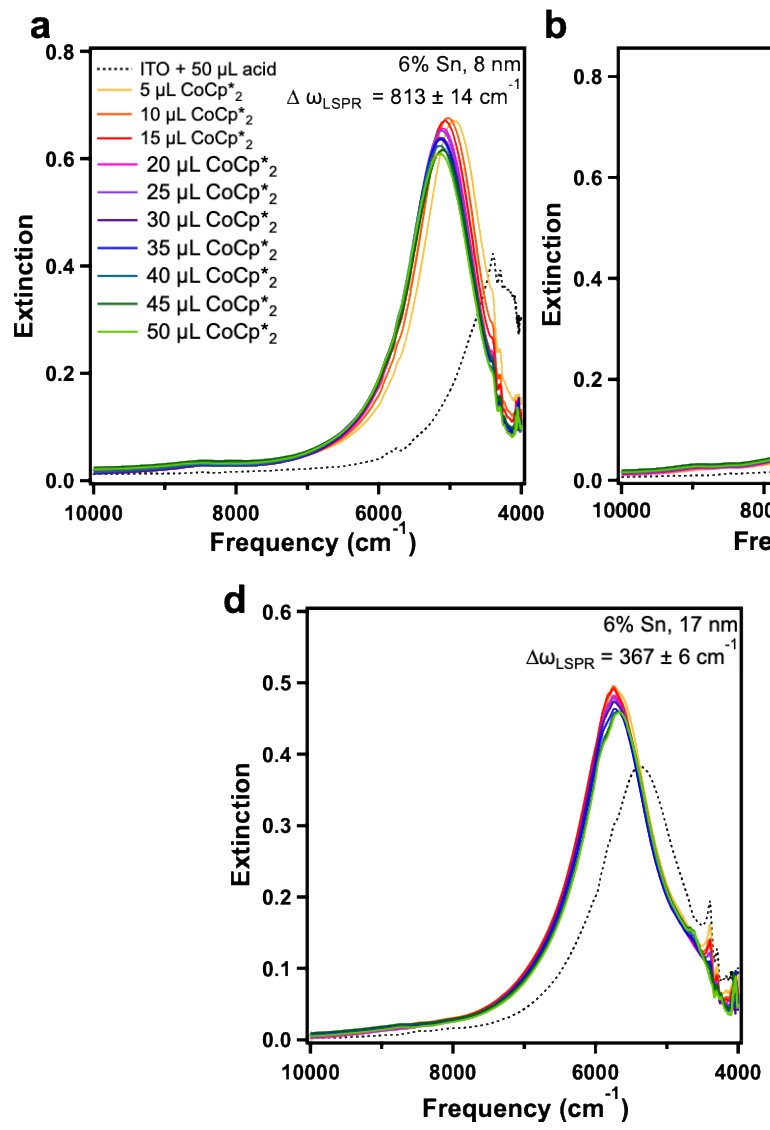

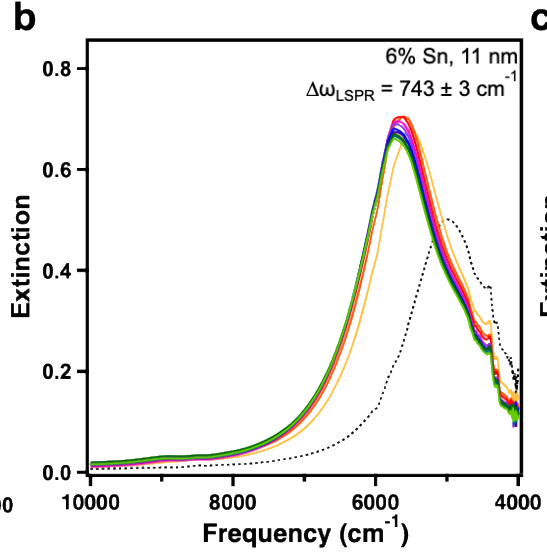

C

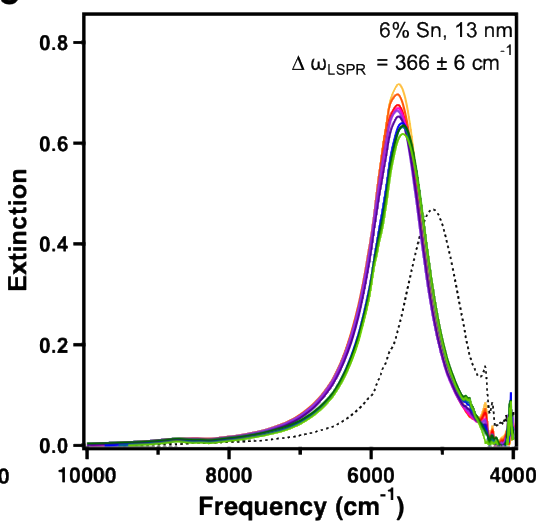

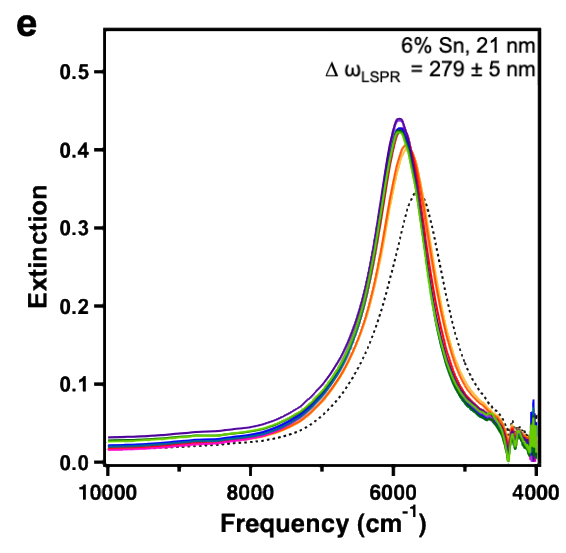

Figure S7: Effect of the addition of chemical reductant $\mathrm{CoCp}_{2}$ on the LSPR extinction spectra of $6 \% \mathrm{Sn}: \mathrm{In}_{2} \mathrm{O}_{3} \mathrm{NCs}$ with different $\mathrm{NC}$ diameters. 

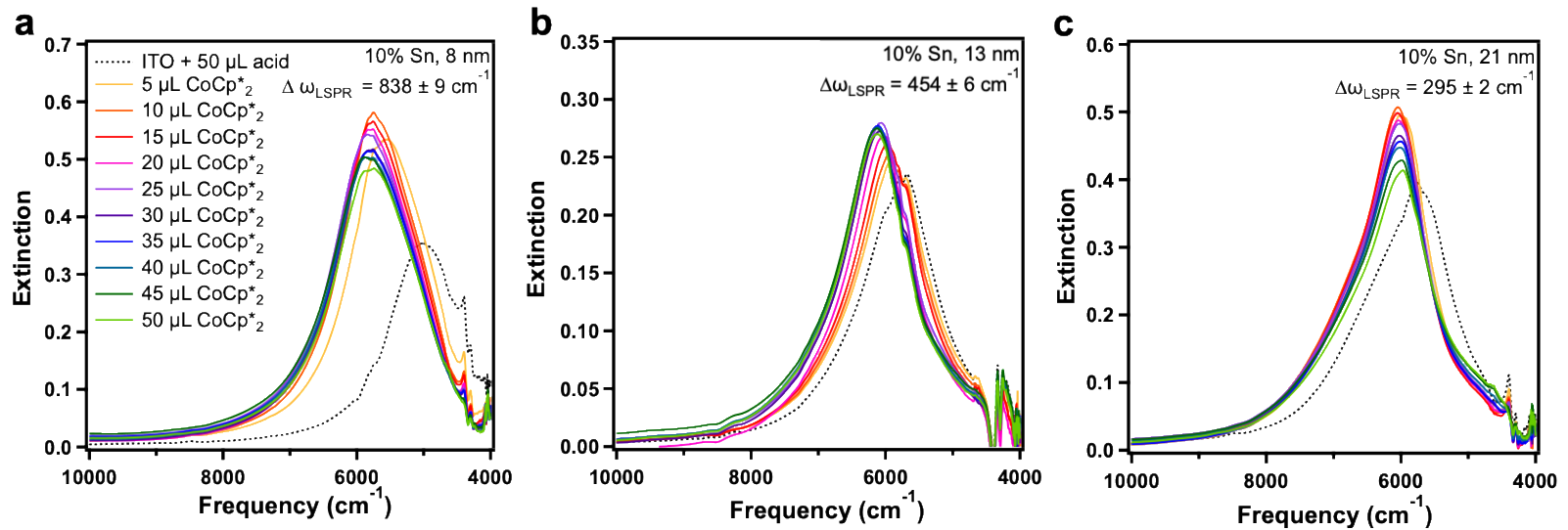

Figure S8: Effect of the addition of chemical reductant CoCp* ${ }_{2}$ on the LSPR extinction spectra of $10 \% \mathrm{Sn}: \mathrm{In}_{2} \mathrm{O}_{3} \mathrm{NCs}$ with different NC diameters.
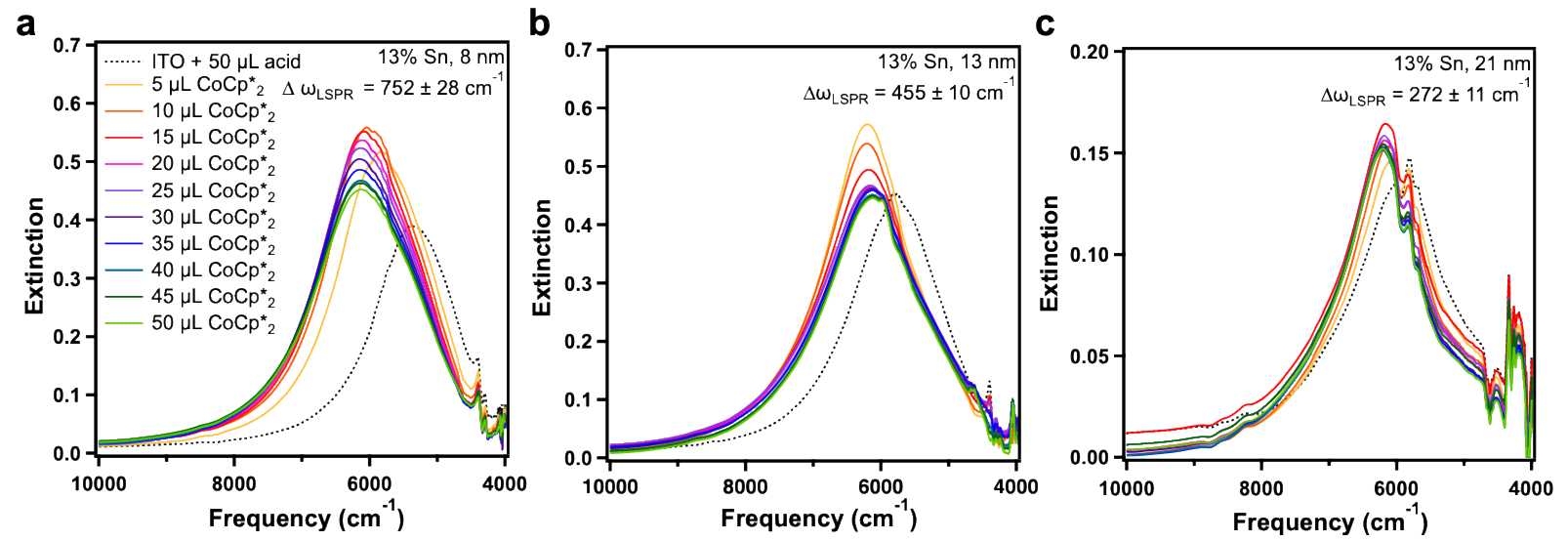

Figure S9: Effect of the addition of chemical reductant CoCp* ${ }_{2}$ on the LSPR extinction spectra of $13 \% \mathrm{Sn}: \mathrm{In}_{2} \mathrm{O}_{3}$ NCs with different NC diameters. 

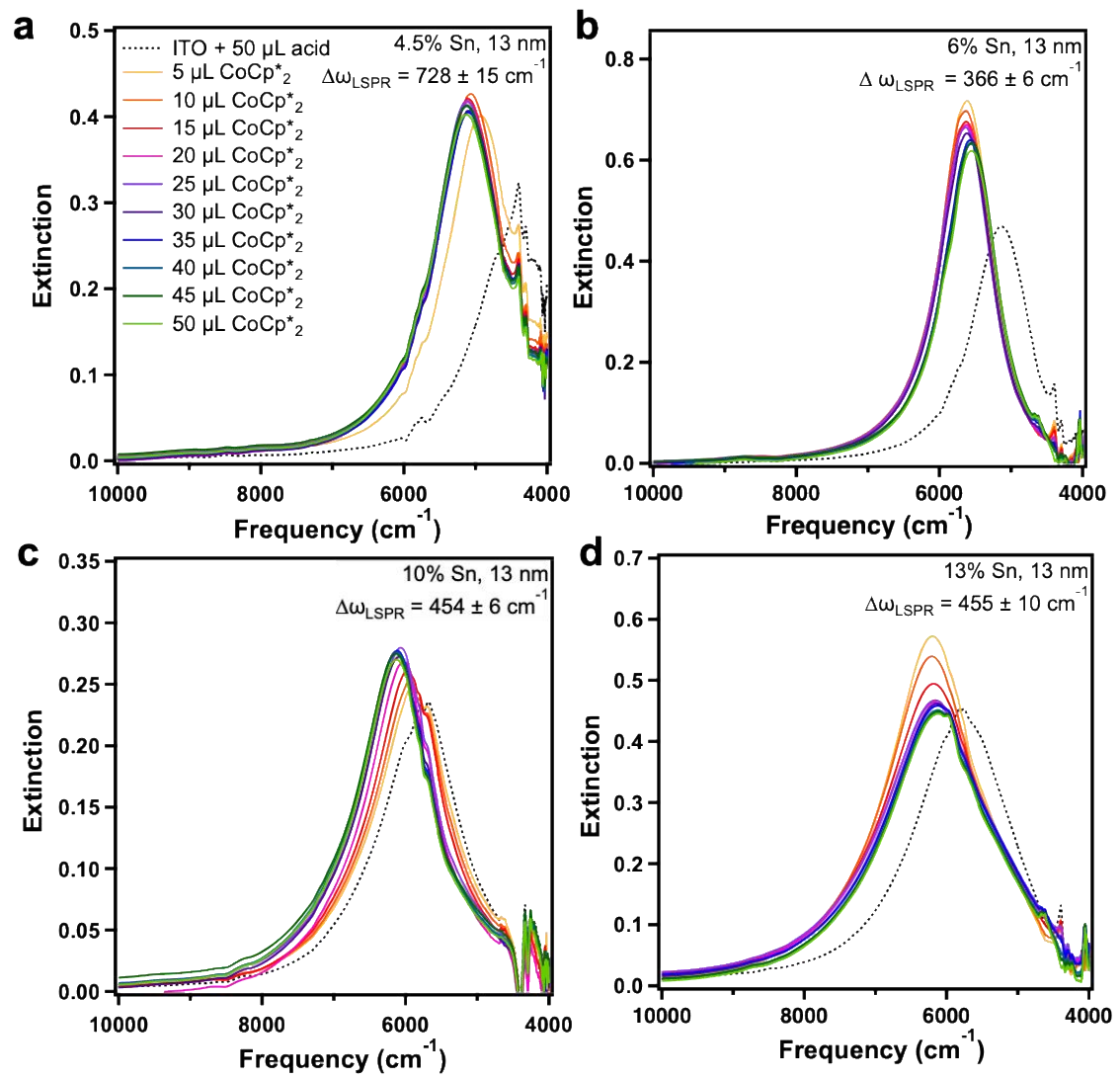

Figure S10: Effect of the addition of chemical reductant $\mathrm{CoCp}_{2}{ }_{2}$ on the LSPR extinction spectra

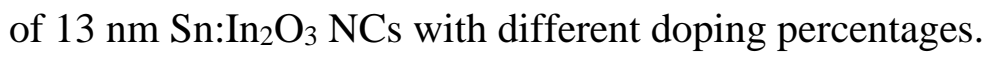



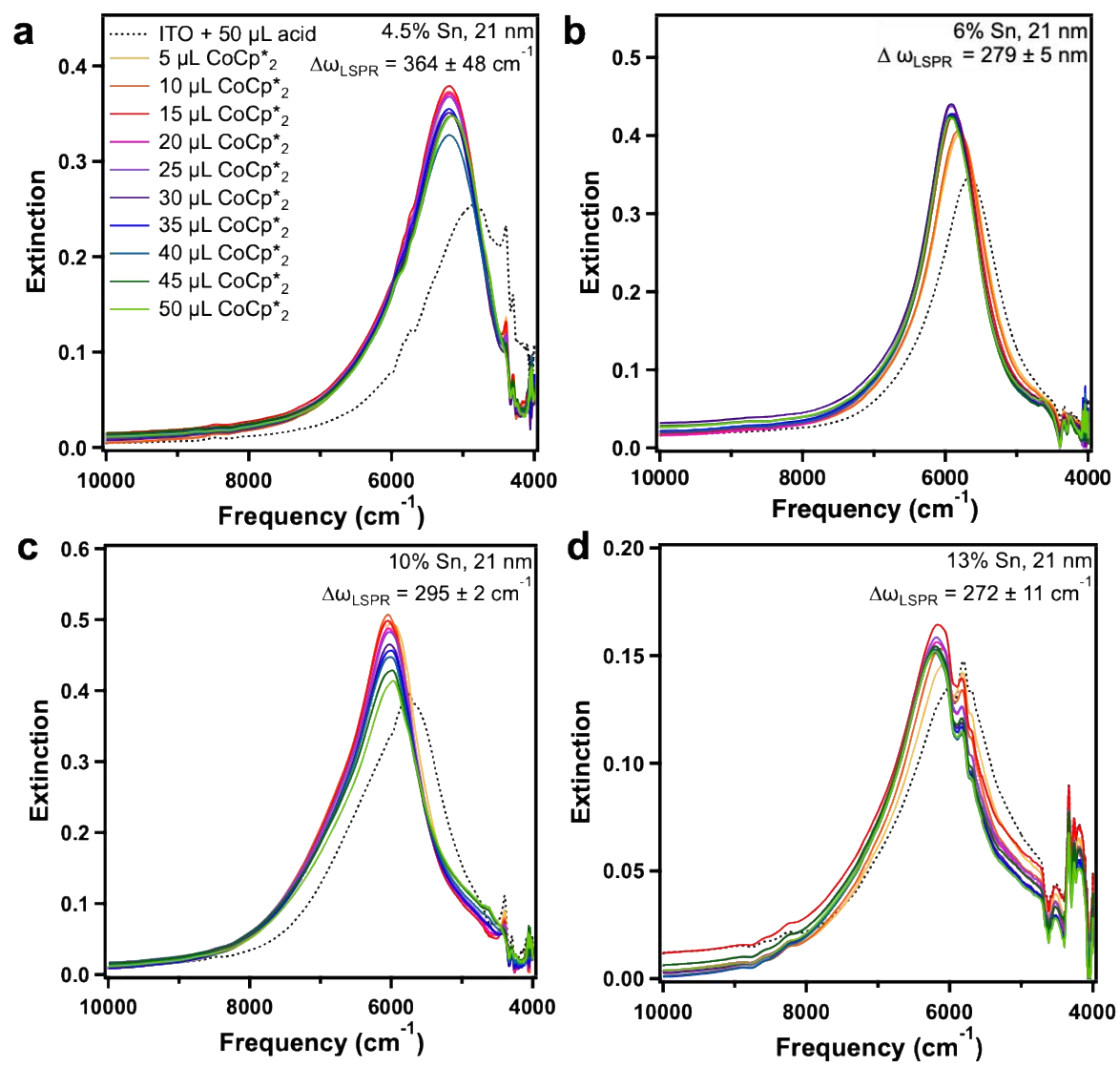

Figure S11: Effect of the addition of chemical reductant $\mathrm{CoCp}_{2}{ }_{2}$ on the LSPR extinction spectra of $21 \mathrm{~nm} \mathrm{Sn}: \mathrm{In}_{2} \mathrm{O}_{3} \mathrm{NCs}$ with different doping percentages. 

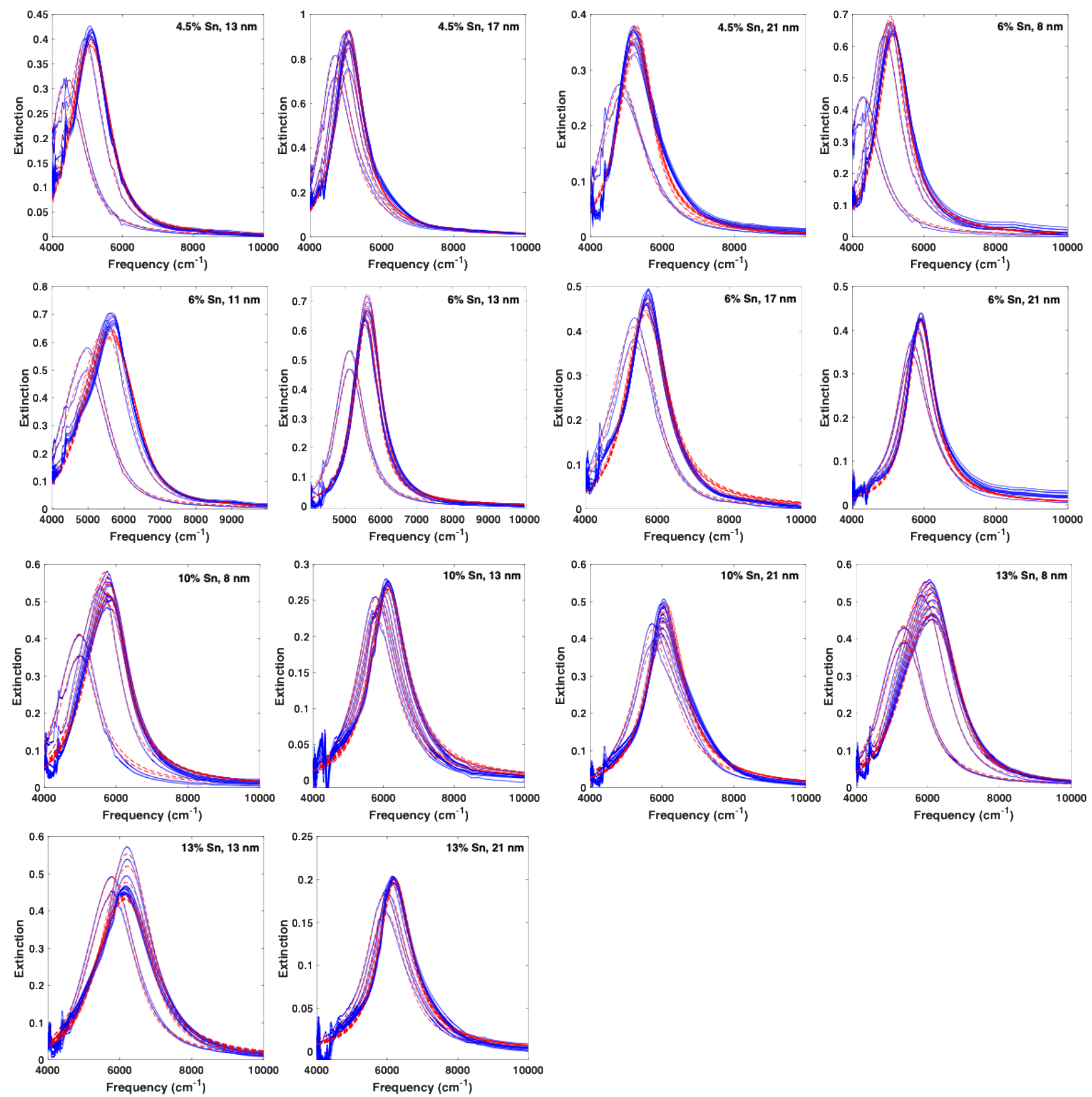

Figure S12: HEDA fits (dashed red) to the experimental LSPR spectra (blue) of $\mathrm{Sn}: \operatorname{In}_{2} \mathrm{O}_{3} \mathrm{NCs}$ belonging to different size and doping series as they evolve during the chemical titration experiment. 

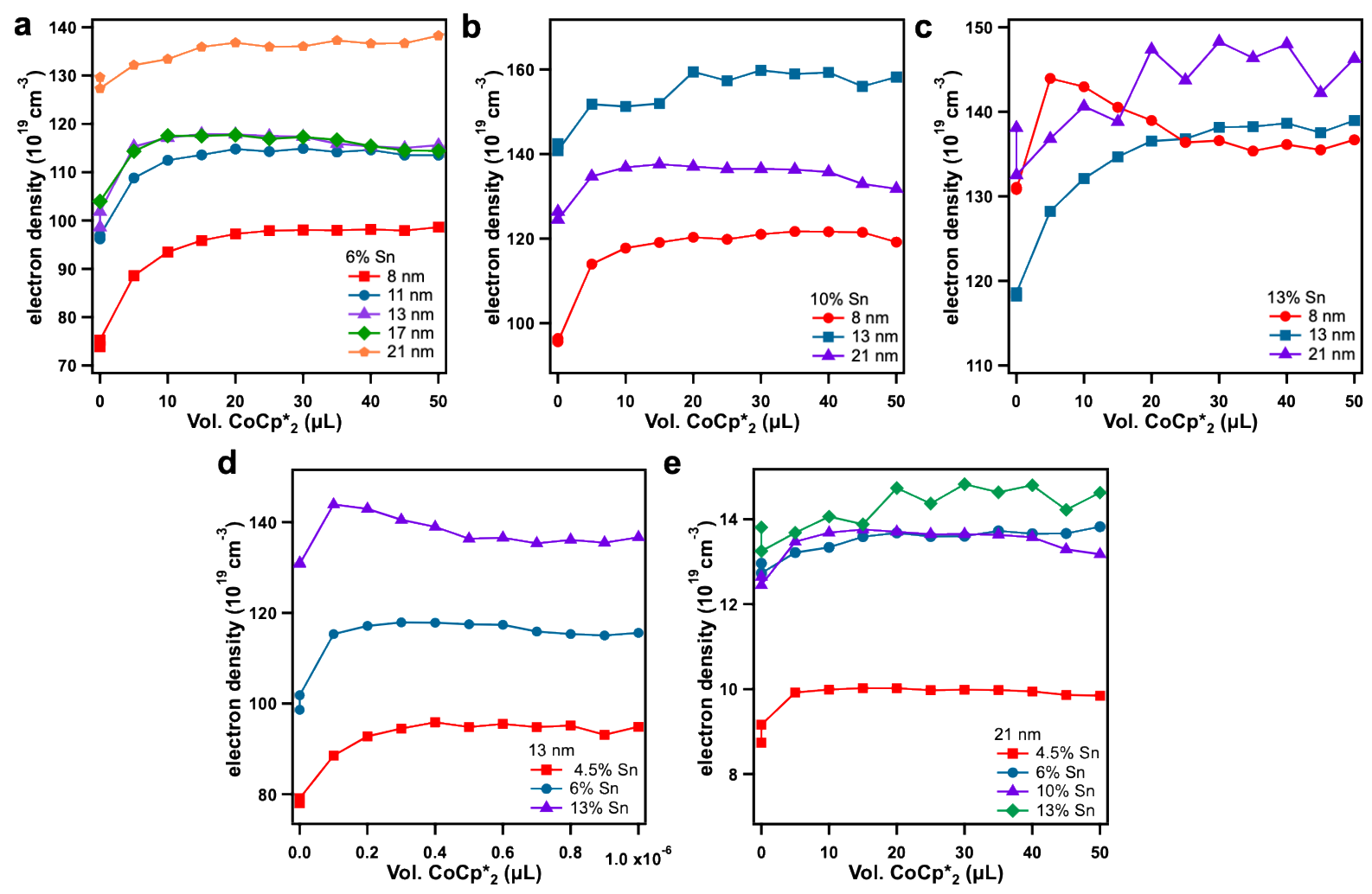

Figure S13: Evolution of electron density of the plasmonic core during the chemical reduction experiment with $\mathrm{CoCp}_{2}{ }_{2}$ for $(\mathrm{a}-\mathrm{c})$ constant doping percentages with different $\mathrm{NC}$ diameter (d-e) constant NC diameter but different doping percentages. Symbols are data points and solid lines are a guide to the eye.

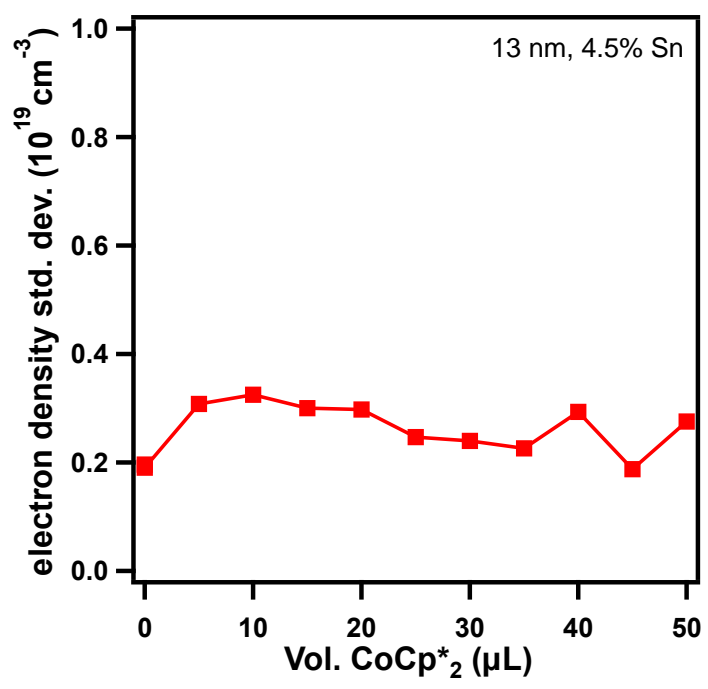

Figure S14: Standard deviation of electron density as obtained by fitting the LSPR with the HEDA model for a $13 \mathrm{~nm}, 4.5 \% \mathrm{Sn}: \mathrm{In}_{2} \mathrm{O}_{3}$ sample. 

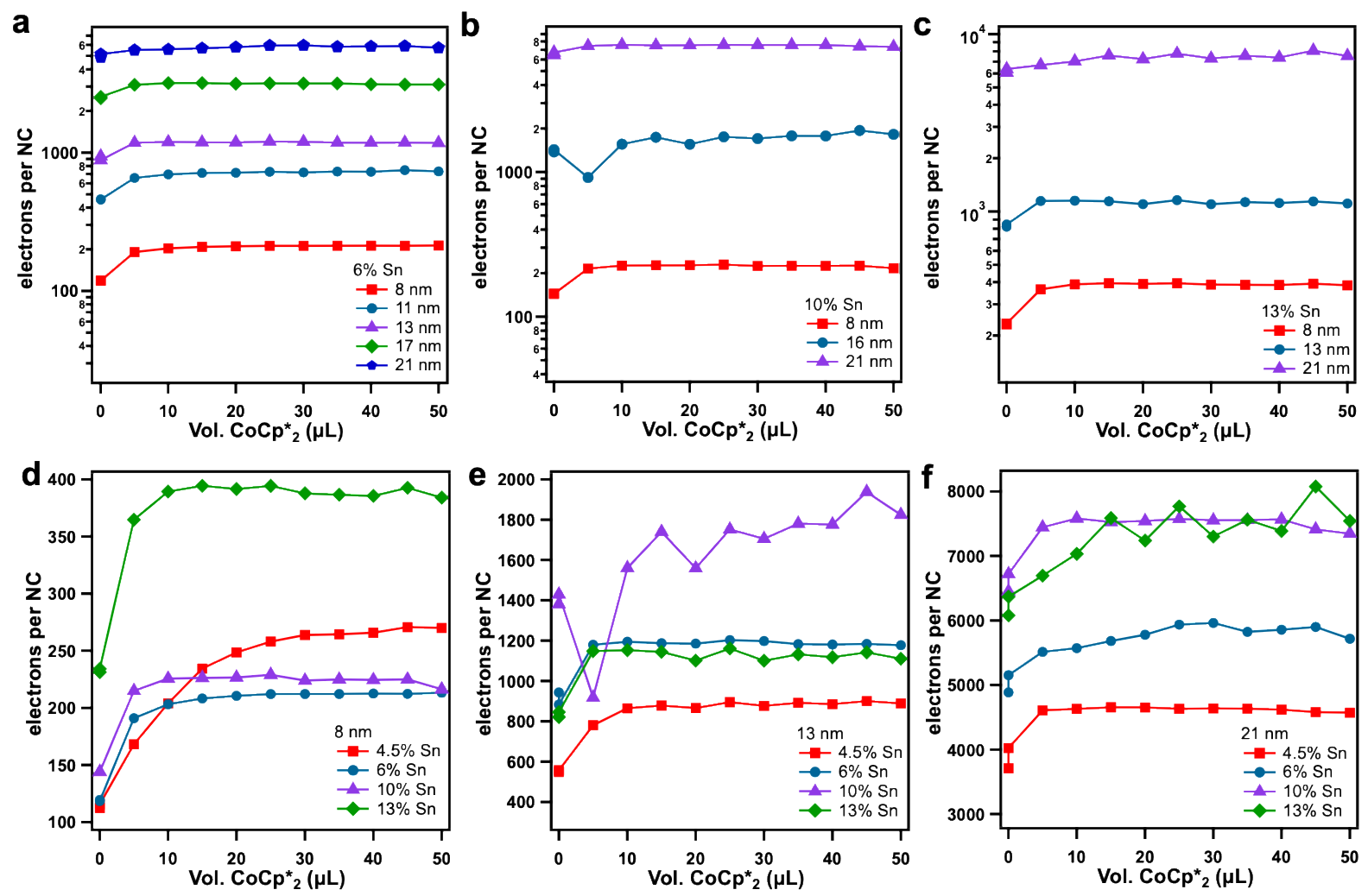

Figure S15: Change in electrons per $\mathrm{NC}$ for $\mathrm{Sn}: \mathrm{In}_{2} \mathrm{O}_{3} \mathrm{NCs}$ with (a-c) constant doping percentages with different $\mathrm{NC}$ diameter (d-f) constant $\mathrm{NC}$ diameter but different doping percentages as $\mathrm{NCs}$ are titrated against $5 \mu \mathrm{L}$ aliquots of $0.02 \mathrm{M} \mathrm{CoCp}^{*}$. Symbols are data points and solid lines are a guide to the eye. 

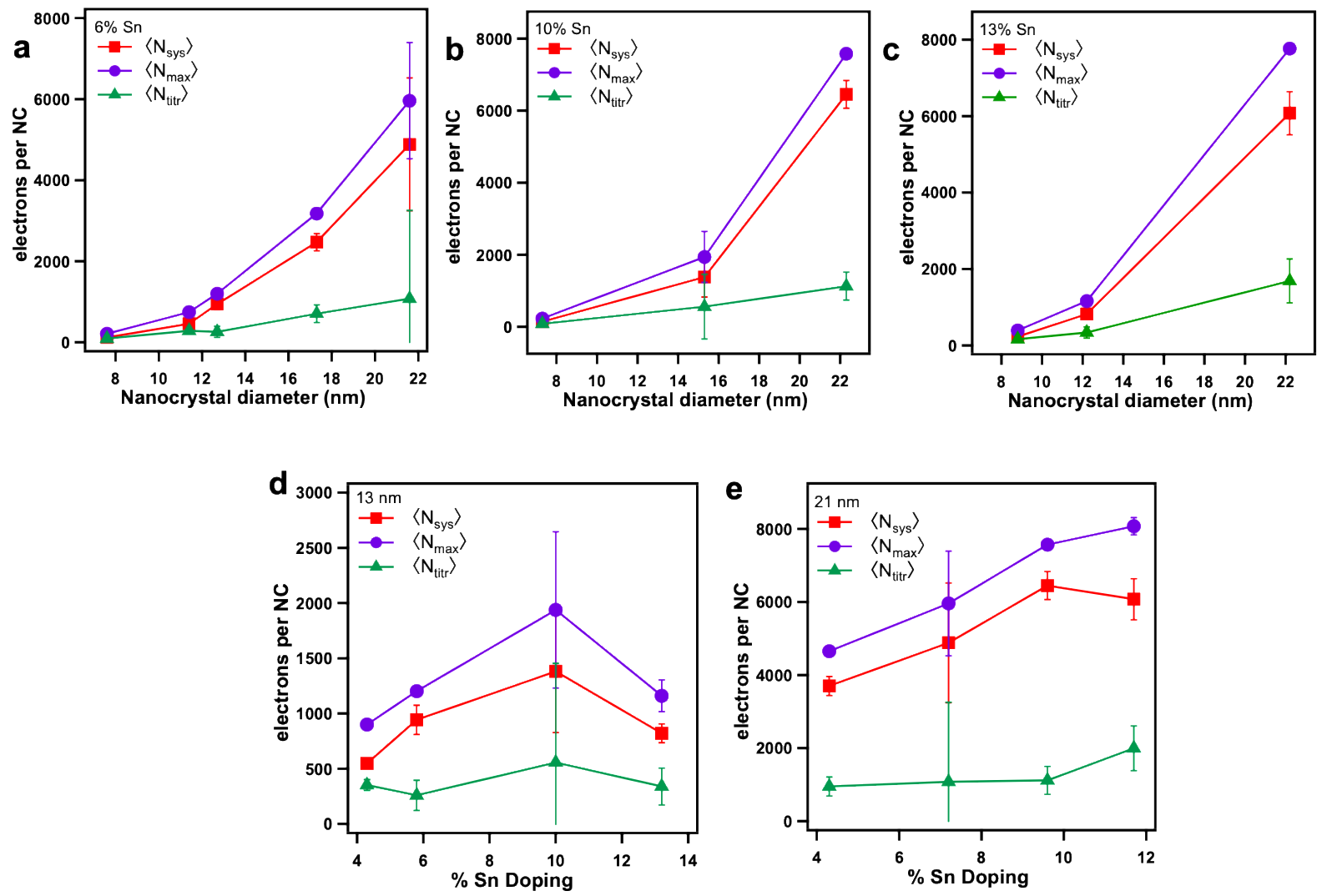

Figure S16: Change in maximum number of electrons per $\mathrm{NC}$ for $\mathrm{Sn}: \mathrm{In}_{2} \mathrm{O}_{3}$ NCs with (a-c) the same doping percentages and different $\mathrm{NC}$ diameter $(\mathrm{d}, \mathrm{e})$ the same $\mathrm{NC}$ diameter but different doping percentages after completion of the chemical reduction experiment. Symbols are data points and solid lines are a guide to the eye.
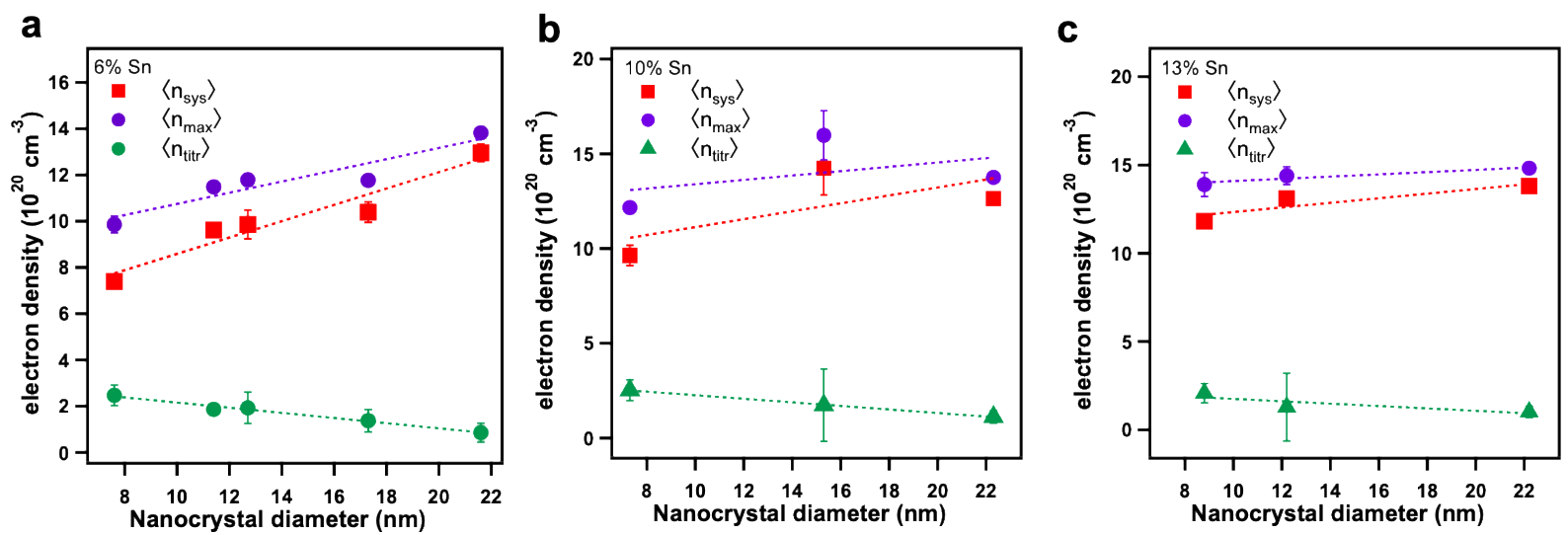

Figure S17: Change in maximum electron density for differently sized $\mathrm{Sn}: \mathrm{In}_{2} \mathrm{O}_{3} \mathrm{NCs}$ with (a) $6 \%$ Sn doping (b) $10 \%$ Sn doping (c) 13\% Sn doping. Symbols are data points and dashed lines are a least-square linear fits to the data points. 

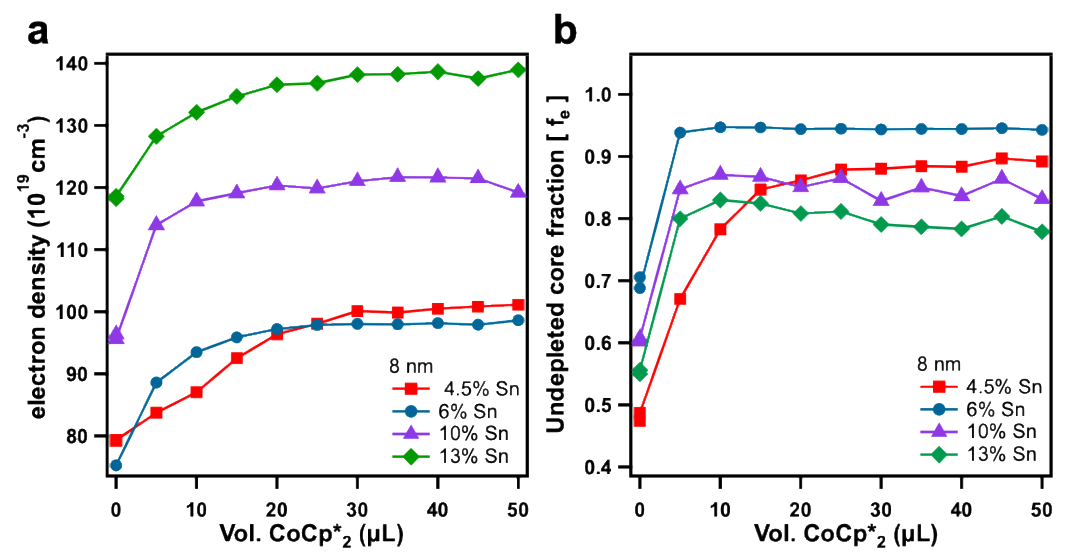

Figure S18: Evolution of (a) electron density (b) volume of the undepleted core during the chemical reduction experiment with CoCp* $*_{2}$ for $8 \mathrm{~nm} \mathrm{Sn}: \mathrm{In}_{2} \mathrm{O}_{3} \mathrm{NCs}$ having different doping percentages. Symbols are data points and solid lines are a guide to the eye.
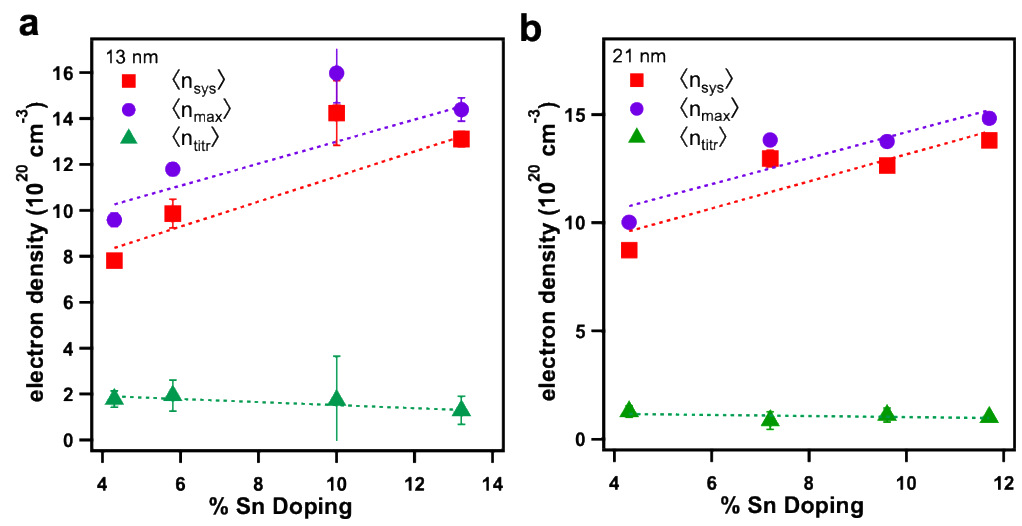

Figure S19: Change in maximum electron density as a function of doping percentage of $\mathrm{Sn}_{2} \operatorname{In}_{2} \mathrm{O}_{3}$ NCs with (a) $13 \mathrm{~nm}$ diameter (b) $21 \mathrm{~nm}$ diameter. Symbols are data points and dashed lines are a least-square linear fits to the data points. 